\title{
Article \\ Alternative Splicing of TaGS3 Differentially Regulates Grain Weight and Size in Bread Wheat
}

\author{
Xiaoli Ren ${ }^{1,2,+}$, Liya Zhi ${ }^{1,2,+}$, Lei Liu ${ }^{3}{ }^{-}$, Deyuan Meng ${ }^{1,2}$, Qiannan Su ${ }^{1}$, Aamana Batool ${ }^{1,2}$, Jun Ji ${ }^{1,4}$, \\ Liqiang Song ${ }^{1,4}$, Na Zhang ${ }^{1,4}$, Lin Guo ${ }^{5}$, Xigang Liu ${ }^{5}{ }^{-}$, Junming $\mathrm{Li}^{1,4,5, *}$ and Wei Zhang ${ }^{6, *}$ \\ 1 Center for Agricultural Resources Research, Institute of Genetics and Developmental Biology, \\ The Innovative Academy of Seed Design, Chinese Academy of Sciences, Shijiazhuang 050022, China; \\ renxiaoli2011@126.com (X.R.); xiaoya_19861028@163.com (L.Z.); mengdeyuan123@163.com (D.M.); \\ SUQIANNAN1923@126.com (Q.S.); aamana.batool@yahoo.com (A.B.); jijun@sjziam.ac.cn (J.J.); \\ lqsong@sjziam.ac.cn (L.S.); zhangna2nina@163.com (N.Z.) \\ 2 The College of Life Science, University of Chinese Academy of Sciences, Beijing 100049, China \\ 3 School of Life Science, Huaiyin Normal University, Huaian 223300, China; leiliu_cell@163.com \\ 4 State Key Laboratory of Plant Cell and Chromosome Engineering, Chinese Academy of Sciences, \\ Beijing 100101, China \\ 5 Ministry of Education Key Laboratory of Molecular and Cellular Biology, Hebei Collaboration Innovation \\ Center for Cell Signaling, Hebei Key Laboratory of Molecular and Cellular Biology, College of Life Sciences, \\ Hebei Normal University, Shijiazhuang 050024, China; linguomail@163.com (L.G.); xgliu@hebtu.edu.cn (X.L.) \\ 6 College of Bioscience and Engineering, Hebei University of Economics and Business, \\ Shijiazhuang 050061, China \\ * Correspondence: ljm@sjziam.ac.cn (J.L.); edithor@126.com (W.Z.) \\ + These authors contributed equally to this work.
}

Citation: Ren, X.; Zhi, L.; Liu, L.; Meng, D.; Su, Q.; Batool, A.; Ji, J.; Song, L.; Zhang, N.; Guo, L.; et al. Alternative Splicing of TaGS3 Differentially Regulates Grain Weight and Size in Bread Wheat. Int. J. Mol. Sci. 2021, 22, 11692. https://doi.org/ $10.3390 /$ ijms222111692

Academic Editors: Agata Gadaleta, Domenica Nigro and Ilaria Marcotuli

Received: 12 September 2021

Accepted: 25 October 2021

Published: 28 October 2021

Publisher's Note: MDPI stays neutral with regard to jurisdictional claims in published maps and institutional affiliations.

Copyright: (C) 2021 by the authors Licensee MDPI, Basel, Switzerland. This article is an open access article distributed under the terms and conditions of the Creative Commons Attribution (CC BY) license (https:// creativecommons.org/licenses/by/ $4.0 /)$.
Abstract: The heterotrimeric G-protein mediates growth and development by perceiving and transmitting signals in multiple organisms. Alternative splicing (AS), a vital process for regulating gene expression at the post-transcriptional level, plays a significant role in plant adaptation and evolution. Here, we identified five splicing variants of $\mathrm{G}_{\gamma}$ subunit gene TaGS3 (TaGS3.1 to TaGS3.5), which showed expression divergence during wheat polyploidization, and differential function in grain weight and size determination. TaGS3.1 overexpression significantly reduced grain weight by $5.89 \%$ and grain length by $5.04 \%$, while TaGS3.2-3.4 overexpression did not significantly alter grain size compared to wild type. Overexpressing TaGS3.5 significantly increased the grain weight by $5.70 \%$ and grain length by $4.30 \%$. Biochemical assays revealed that TaGS3 isoforms (TaGS3.1-3.4) with an intact OSR domain interact with WGB1 to form active $G_{\beta \gamma}$ heterodimers that further interact with WGA1 to form inactive $G_{\alpha \beta \gamma}$ heterotrimers. Truncated isoforms TaGS3.2-3.4, which lack the C-terminal Cys-rich region but have enhanced binding affinity to WGB1, antagonistically compete with TaGS3.1 to bind WGB1, while TaGS3.5 with an incomplete OSR domain does not interact with WGB1. Taking these observations together, we proposed that TaGS3 differentially regulates grain size via AS, providing a strategy by which the grain size is fine-tuned and regulated at the post-transcriptional level.

Keywords: bread wheat; TaGS3; alternative splicing; grain weight; grain size

\section{Introduction}

Bread wheat (Triticum aestivum L.) is a global staple crop. High yield, as the prime breeding target in cereals, is determined by the panicles per plant, grain number per spike, and grain weight. Grain weight is the major determinant of yield potential, and it is largely dependent on grain size. Thus, grain size has long been the critical target of selection in wheat breeding [1].

Genetic and molecular analyses have identified numerous genes involved in multiple signaling pathways that regulate grain size [2]. One of the most important pathways is 
the G-protein signaling pathway. Heterotrimeric G-proteins, comprising $\mathrm{G}_{\alpha}, \mathrm{G}_{\beta}$, and $\mathrm{G}_{\gamma}$ subunits, are the molecular switches that transmit signals from transmembrane receptors to downstream target proteins. $G_{\gamma}$ binds tightly to $G_{\beta}$, forming a functional $G_{\beta \gamma}$ unit that can only be dissociated under denaturing conditions [3]. The structural diversity of $\mathrm{G}_{\gamma}$ subunits solely provides functional selectivity to the heterotrimer [4]. $G_{\gamma}$ subunits are divided into three types according to their C-terminal structures, of which the atypical $\mathrm{G}_{\gamma}$ subunit both contains a plant-specific organ size regulation (OSR) domain in the $\mathrm{N}$ terminus, and possesses a C-terminal Cys-rich region [5].

One noncanonical $G_{\gamma}$ subunit (AGG3) is a positive regulator of seed size in Arabidopsis [6] because the null agg3 mutant produces smaller seeds and organs than those of wild type (WT), but AGG3 overexpression lines generate larger seeds and organs. OsGS3, a $\mathrm{G}_{\gamma}$ subunit in rice, acts as a negative regulator of grain weight and length [7]. In de novo domestication, GS3 sgRNA targeting significantly increased grain length in PPR1 mutants of wild allotetraploid rice [8]. A mutant with an allelic variation resulting in the truncated OSR domain of OsGS3 produces long grains [9], whereas the OsGS3 mutant with deletion of the C-terminal Cys-rich region produces very short grains [10], indicating the divergent functions of the N-terminal OSR domain and the C-terminal Cys-rich region of OsGS3 in regulating rice grain size. In wheat, three $T a G S 3$ homeologous genes were identified on chromosomes 7A, 4A and 7D (designed as TaGS3-7A, TaGS3-4A and TaGS3-7D), which negatively regulate grain weight and size [11]. Although multiple haplotypes in TaGS3 homeologs have been identified, the underlying molecular mechanisms determining the existence of these multiple haplotypes are still unclear.

Alternative splicing (AS) is a key post-transcriptional regulatory mechanism for expanding proteomic diversity and functional complexity [12], even generating trait diversity [13]. AS mainly results in generation of an alternative $3^{\prime}$ or $5^{\prime}$ splicing site (Alt $3^{\prime}$ ss and Alt $5^{\prime}$ ss, respectively), ) intron retention (IR), and exon skipping (ES) [13,14]. AS can produce truncated proteins, disrupting key domains or abolishing interaction with other proteins and thereby preventing the formation of functional protein complexes [15,16]. Moreover, some splicing variants can compete with a constitutive variant and interfere with its function in a dominant negative manner $[17,18]$. In our previous study [11], we observed the expression of multiple similar transcripts during TaGS3 cloning. The differences between the TaGS3 transcripts and their biological functions were unclear.

AS participates in the regulation of a series of grain-related traits. A sequence duplication at exon 2 of $P s y-A 1$ creates a new splice site and causes AS with an activated cryptic exon, resulting in four splicing variants. Only the constitutive splicing variant produces an enzymatically active protein, and its mRNA abundance is reduced by titration with the other splicing variants, which is argued to result in a reduction in PSY and thus carotenoid content in wheat [19]. Two mRNA variants are found for Delay of Germination1 (DOG1), lgDOG1 and shDOG1. shDOG1 is translated and functional, in contrast to lgDOG1, promoting seed dormancy in Arabidopsis [20]. AS of several genes was reported to affect rice grain size. OsLG3b encodes MADS-box transcription factor1 (OsMADS1). Its six SNP variations lead to AS, introducing a premature termination codon (PTC), and resulting in the truncation of 32 amino acid residues that are positively associated with grain length [21]. OrMKK3 undergoes AS and produces splicing variants. The overexpression of four of the five OrMKK3 splicing variants results in reduced grain length and width [22]. Nevertheless, whether and how these splicing variants interact with each other to modulate agronomic traits remain unknown.

Bread wheat has a huge and complex genome with a number of genes playing complex roles [23]. The genome-wide profiling of AS revealed a complex AS landscape in wheat, showing that approximately $22.1 \%$ of genes exhibit AS [24]. AS dynamics and evolutionary divergence during embryogenesis in wheat species indicated that AS provides opportunities for transcriptional and proteomic plasticity, and the potential for generating trait diversity [12]. Some reports have illustrated that AS events in wheat are substantially related to grain biochemical traits such as phytoene synthase activity [19], polyphenol 
oxidase activity [25], and starch synthase activity [26]. AS is also involved in disease resistance [27] and abiotic stresses tolerance [28-30]. Studies related to AS on wheat grain traits are mainly focused on grain quality. AS events and their biological functions in wheat genes related to grain weight and size remain poorly understood.

Here, we report that TaGS3 undergoes AS and produces five splicing variants, corresponding to the constitutive TaGS3.1, the truncated TaGS3.2-3.4 lacking the C-terminal Cys-rich region, and the truncated TaGS3.5 that contains the disrupted OSR domain. Evolutionary analysis showed that AS of GS3 did not emerge during the process of wheat polyploidization, but pre-existed in the ancestor species of Triticeae crops. Given proteinprotein interactions between TaGS3 isoforms and WGB1 $\left(\mathrm{G}_{\beta}\right)$, we biochemically analyzed their nature and found that TaGS3.2, TaGS3.3, and TaGS3.4 interfere with TaGS3.1 function by competitively forming functional $\mathrm{G}_{\beta \gamma}$ heterodimers, and that TaGS3.5 is unable to form a $G_{\beta \gamma}$ heterodimer with WGB1. Correspondingly, overexpression of the five TaGS3 splicing variants in bread wheat exhibited divergent effects on grain development. In particular, TaGS3.5 overexpression produced significantly larger and heavier grains. Taken together, these observations support the proposal that the TaGS3-mediated signal transduction pathway regulates grain weight and size via AS, providing a strategy by which the regulation of grain size is fine-tuned and balanced at the post-transcriptional level.

\section{Results}

\subsection{AS Occurrence of TaGS3 in Wheat}

A previous study indicated that TaGS3 negatively regulates grain weight and size in wheat [10]. During TaGS3 cloning, we identified several distinct CDSs of TaGS3. To decipher the identity of the transcripts, we sequenced the amplicons and confirmed the presence of five unique TaGS3 transcripts (Figure S1). Except for the constitutive variant TaGS3.1, four splicing variants arise from Alt $3^{\prime} \mathrm{ss}$, Alt $5^{\prime}$ ss, IR, and ES, leading to the production of TaGS3.2, TaGS3.3, TaGS3.4, and TaGS3.5, respectively (Figure 1A).

TaGS3.1 contains five exons and four introns, and is predicted to encode the full length $\gamma$-subunit protein of 170 amino acid residues, as previously reported [11]. Sequence analysis revealed that AS results in TaGS3.2, TaGS3.3, and TaGS3.4 with varied degrees of retention in the third intron, with TaGS3.2 harboring seven nucleotides (CTTGCAC) at the $3^{\prime}$-end of intron 3, TaGS3.3 harboring 14 nucleotides (GTATGGATTTTCAG) at the $5^{\prime}$-end of intron 3 , and TaGS3.4 harboring the full length of intron 3 (82 bp). As a result, TaGS3.2, TaGS3.3, and TaGS3.4 are all frame-shifted, with introduction of a PTC at the 213th nucleotide site of exon 4, 285th nucleotide site of exon 5, and 216th nucleotide site of exon 4, respectively. For TaGS3.5, AS has resulted in skipping the third exon (Figure 1A and Figure S2).

Homology comparison shows $88.36 \%$ and $42.92 \%$ similarities in the $\mathrm{N}$ terminus and C terminus of GS3 in T. aestivum, H. vulgare, S. italica, S. bicolor (L.) Moench, Z. mays, P. hallii, $O$. sativa, and B. distachyon (Figure S3), indicating the high degree of conservation in the $\mathrm{N}$ terminus of GS3 homologs. The C-terminal Cys-rich region of GS3, however, is mostly divergent in length and composition in Poaceae species. Rice OsGS3 possesses a conserved OSR domain (7-72 aa), sharing high similarity with the N-terminal regions of $G_{\gamma}$ in a number of species, ranging from angiosperm to gymnosperm [10]. Alignment analysis of TaGS3.1 and OsGS3 revealed the presence of conserved domains in TaGS3.1: one OSR domain (1-66 aa), two transmembrane domains (87-104 and 127-158 aa) and one Cys-rich region (67-170 aa) (Figure 1B and Figure S4A). The intron retention of TaGS3.2, TaGS3.3, and TaGS3.4 results in the 70, 94, and 71 aa truncation of TaGS3, respectively, leading to deletion of the $\mathrm{C}$-terminal Cys-rich region containing two predicted transmembrane domains (Figure S4B-D). In addition, TaGS3.2 contains an intact OSR domain (1-66 aa), and the OSR domains in TaGS3.3 and TaGS3.4 show 1 aa difference (Figure 1B). The skipping of the third exon ( $45 \mathrm{bp}$ ) of TaGS3.5 does not lead to a frame shift, and the predicted encoded peptide is 155 amino acid residues. This truncated protein lacks 15 amino acid residues, including the DPFITI motif at the 52nd amino acid residue site in the OSR domain (Figure 1C). Therefore, TaGS3.5 is predicted to contain one incomplete OSR domain 
(1-51 aa) and two complete predicted transmembrane domains (72-89 aa, 112-143 aa) (https:/ / embnet.vital-it.ch/software/TMPRED_form.html accessed on 15 August 2021) (Figure 1B and Figure S4E).

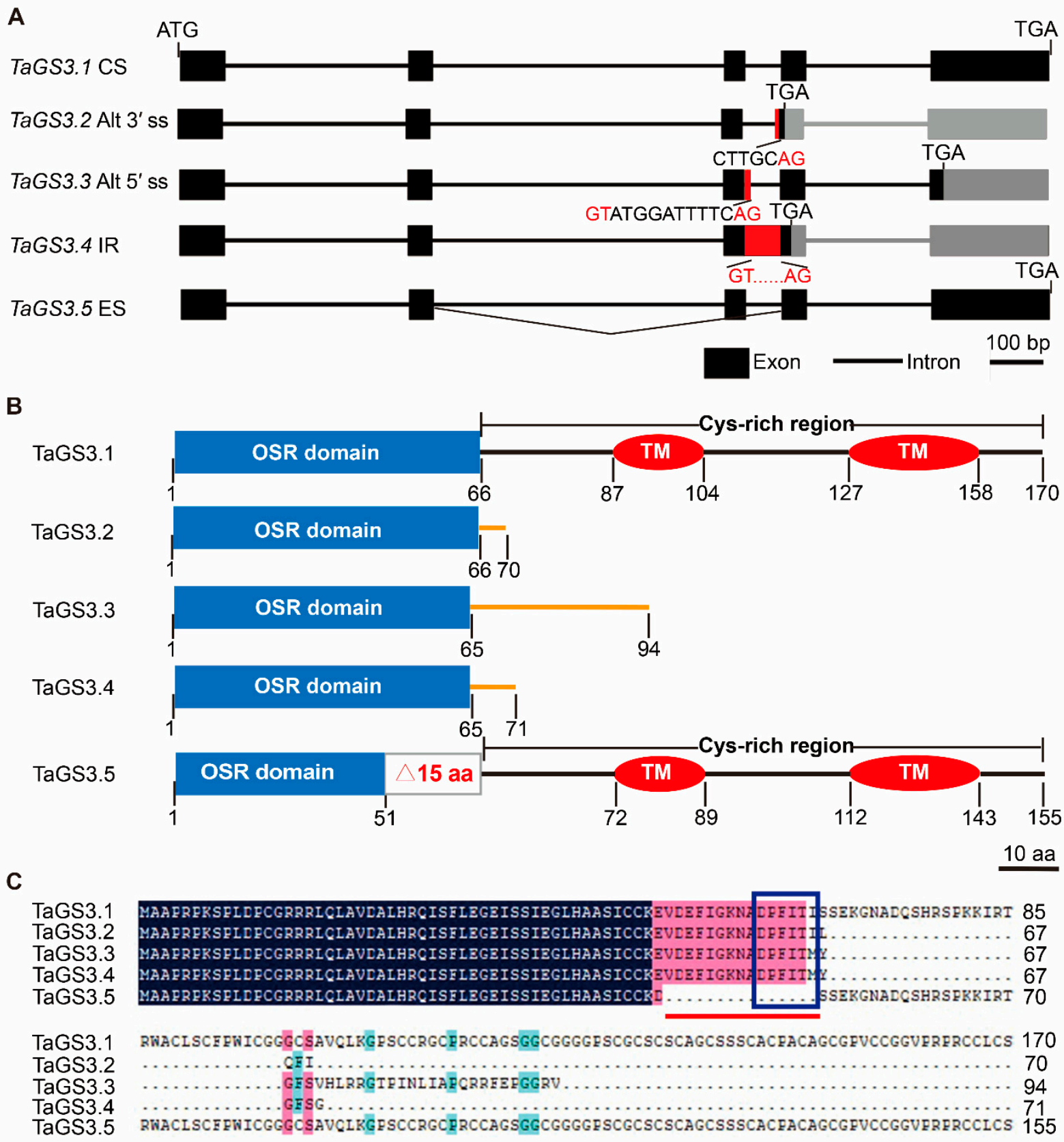

Figure 1. TaGS3 is subject to AS.(A) Schematic representation of gene structures of TaGS3 splicing variants. CS, constitutive splicing; Alt $3^{\prime}$ ss, alternative $3^{\prime}$ splice site; Alt $55^{\prime}$ ss, alternative $5^{\prime}$ splice site; IR, intron retention; ES, exon skipping. Gene exons and introns are shown by black boxes and lines, respectively. TaGS3 splicing variants are distinguished by red boxes (intron 3 retention section) and polyline (exon 3 skipping). GT ... AG represents the canonical splicing site. Bar $=100 \mathrm{bp}$. (B) The structural diagrammatic sketch of the five TaGS3 isoforms. Bar $=10$ aa. OSR, organ size regulation domain; TM, putative transmembrane domain. The orange lines indicate the C-terminal non-Cys-rich region. (C) Protein sequence alignment of the TaGS3 isoforms. The 15 missing amino acid residues of TaGS3.5 are highlighted by red line. The DPFITI motif is marked by a blue quadrilateral. 


\subsection{GS3 Splicing Variants in Wheat Species}

To determine whether AS patterns changed during wheat polyploidization, we examined three diploid wheat progenitor species, namely, TMB02 (T. boeoticum, AA), TH02 (Ae. sharonensis, SS), and Y199 (Ae. tauschii, DD), and two T. aestivum cv. KN9204 and KN199, which showed divergent grain morphological variations and relative GS3 expression levels (Figure 2A,B). Analyses on the occurrence frequency of the five GS3 splicing variants in developing grains showed that GS3.1 is the major splicing variant, representing $92.45 \%, 91.94 \%$, and $92.27 \%$ in diploid TMB02, TH02, and $\mathrm{Y} 199$ and $97.52 \%$ and $96.34 \%$ in hexaploid KN9204 and KN199, respectively. The total ratio of the four other splicing variants accounted for $7.55 \%, 8.06 \%, 7.73 \%, 2.48 \%$, and 3.66\% in TMB02, TH02, Y199, KN9204, and KN199, respectively (Figure 2C).

A

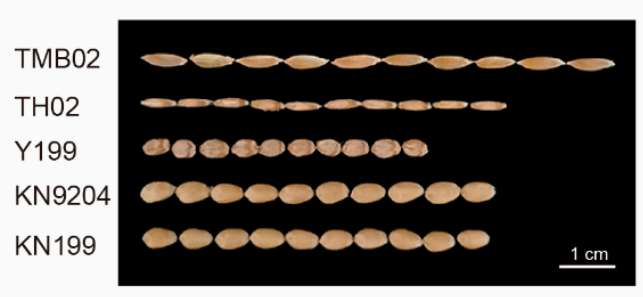

C
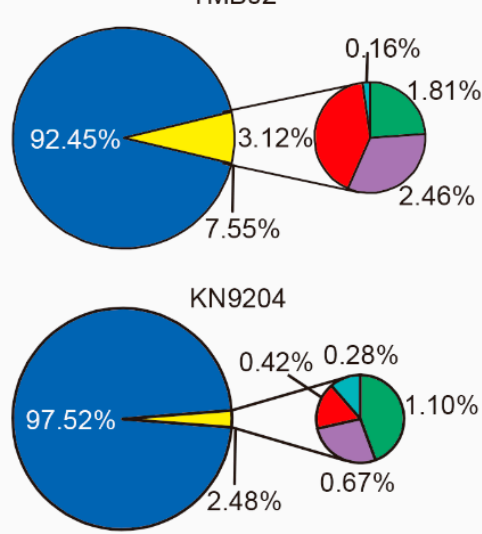

B

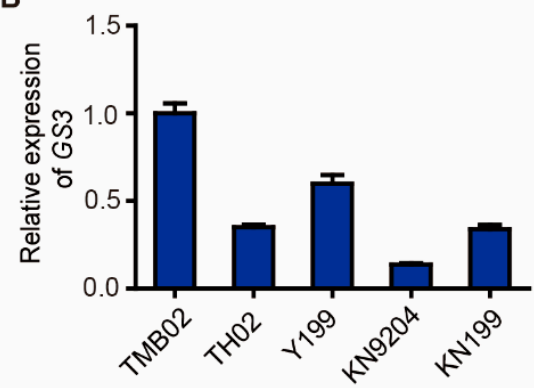

TH02

Y199
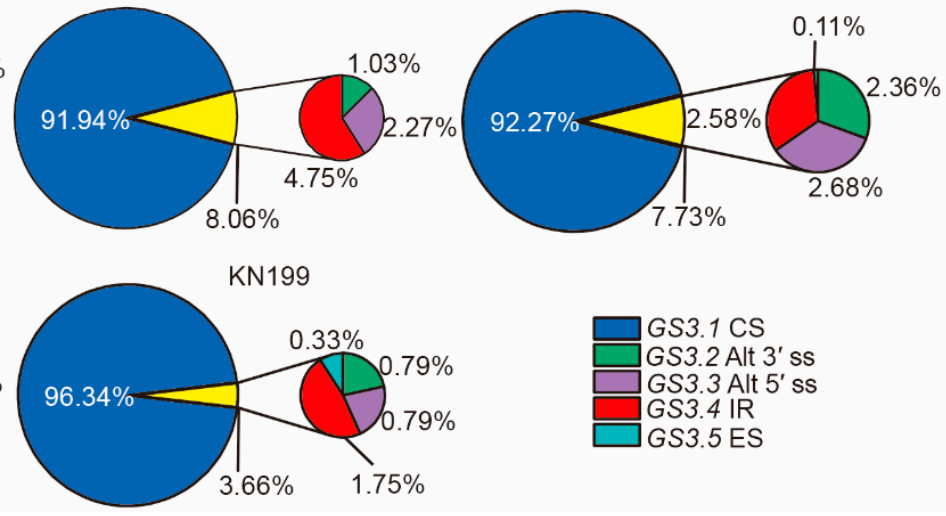

GS3.1 CS

GS3.2 Alt 3' ss

GS3.3 Alt 5' ss

GS3.4 IR

GS3.5 ES

Figure 2. The occurrence frequency of GS3 splicing variants in diploid wheat progenitors and hexaploid wheat. (A) Phenotypic comparison of grain traits among the indicated diploid wheat progenitors and hexaploid wheat cultivars. Bar $=1 \mathrm{~cm}$. (B) Relative expression of GS3 in mature seeds of the indicated diploid wheat progenitors and hexaploid wheat cultivars. Normalized expression values of GS3 splicing variants relative to GAPDH were given as mean \pm SEM from three replicates. (C) The occurrence frequency of GS3 splicing variants in developing grains of the indicated diploid wheat progenitors and hexaploid wheat cultivars. The GS3 splicing variants and AS types are represented by different colors (dark blue, green, purple, red, and light blue stand for GS3.1 CS, GS3.2 Alt 3' ss, GS3.3 Alt 5' ss, GS3.4 IR, and GS3.5 ES, respectively).

Except for constitutive splicing variant GS3.1, the distributions of the four other GS3 splicing variants were analyzed in diploid and hexaploid species. GS3.4 is the most abundant splicing variant in TMB02 and TH02, followed by GS3.3, GS3.2, and GS3.5. There is no obvious difference in the proportion of GS3.2, GS3.3, and GS3.4 in Y199, accounting for $2.36 \%, 2.68 \%$, and $2.57 \%$, respectively. In KN9204, GS3.2 is the most abundant splicing variant, followed by GS3.3, GS3.4 and GS3.5. In KN199, the distribution of GS3 splicing variants is similar to that in TMB02 and TH02, with GS3.4 being the most abundant splicing variant (Figure 2C). Taken together, GS3.1, GS3.2, GS3.3, and GS3.4 variants are conserved in diploid species, while GS3.5 shows divergent patterns, with a small portion of GS3.5 
transcript identified in TMB02 (0.16\%) and Y199 (0.11\%) but not in TH02. Moreover, TaGS3 exhibited different splicing patterns among the triplex in hexaploid wheat. GS3.1, GS3.3, and GS3.4 variants were all examined in the TaGS3 triplex; GS3.2 was only identified from TaGS3-4A and TaGS3-7D, which is attributed to the absence of one canonical splice junction (AG) in the third intron of TaGS3-7A compared with those of TaGS3-4A and TaGS3-7D (Figure S5). GS3.5 was identified from TaGS3-7A and TaGS3-4A in KN9204, but from TaGS3-4A and TaGS3-7D in KN199 (Table S1).

\subsection{Expression Patterns and Subcellular Localization of TaGS3 Splicing Variants}

The temporal and spatial expression patterns of the five splicing variants of TaGS3 were investigated in KN9204 through qRT-PCR using cDNA-specific primers. TaGS3.1, TaGS3.2, TaGS3.3, and TaGS3.4 were ubiquitously expressed with similar patterns in various wheat tissues at different developmental stages, showing higher expression levels in young spikes and developing grains than in the roots, stems, and leaves. Nevertheless, there were notable differences in expression abundances among the 5 variants; the maximum expression levels of TaGS3.1, TaGS3.2, and TaGS3.3 were observed in the 21 DPA (day post-anthesis) grains, TaGS3.4 expression level peaked in the 28 DPA grains (Figure 3A), but TaGS3.5 expression was undetectable in all assayed tissues by qRT-PCR.

AS frequently results in alterations in protein structures and subcellular locations [31]. To determine the subcellular localization of TaGS3 isoforms, we expressed the five TaGS3 splicing variants as fusions with GFP under the control of the CaMV 355 promoter in $N$. benthamiana. The localization of TaGS3.1-GFP and TaGS3.5-GFP fusion protein fluorescence was only observed in the plasma membrane, while TaGS3.2-GFP, TaGS3.3-GFP, and TaGS3.4-GFP fusion protein fluorescence was observed in both the plasma membrane and the nuclei (Figure 3B). Consistent with these results, Arabidopsis AGG3 was present in the plasma membrane, but AGG3 ${ }^{\triangle 108-125}$, in which the predicted transmembrane domain is deleted, was observed in both the plasma membrane and the nucleus [6].

\subsection{Overexpression of TaGS3 Splicing Variants Confers Different Effects on Wheat Grain Weight and Size}

To study the genetic effects of the five TaGS3 splicing variants in bread wheat, we generated TaGS3.1, TaGS3.2, TaGS3.3, TaGS3.4, and TaGS3.5 transgenic lines in T. aestivum cv. KN199 (Figure 4A). Fold increase in the expression abundances of TaGS3 variants was examined in $\mathrm{T}_{3}$ homozygous overexpression lines (Figure S6). To investigate the relative abundance of the specific proteins, we tested the transgenic plants expressing GFP tagged-fusion of TaGS3.1, TaGS3.2, TaGS3.3, TaGS3.4, and TaGS3.5 in KN199 driven by the ubiquitin promoter. Western blotting analysis showed that TaGS3.1-GFP, TaGS3.2-GFP, TaGS3.3-GFP, TaGS3.4-GFP, and TaGS3.5-GFP were all overexpressed in the transgenic bread wheat (Figure $4 \mathrm{~B}$ ).

The stability of TaGS3 isoforms in the corresponding overexpression lines was assayed. Immunoblot analysis revealed trace expression of TaGS3.2-GFP that was barely detected, and the abundance of TaGS3.3-GFP and TaGS3.4-GFP was significantly reduced under the treatment of cycloheximide $(\mathrm{CHX})$, a protein synthesis inhibitor, whereas the accumulation of TaGS3.1-GFP and TaGS3.5-GFP only changed slightly. The accumulation of all TaGS3.2-GFP, TaGS3.3-GFP, and TaGS3.4-GFP but not TaGS3.1-GFP and TaGS3.5-GFP increased under treatment with the proteasome inhibitor MG132 (Figure S7). These results indicate that TaGS3.1 and TaGS3.5 are more stable than TaGS3.2-3.4, implying the strong influence of the C-terminal Cys-rich region on TaGS3's stability. 

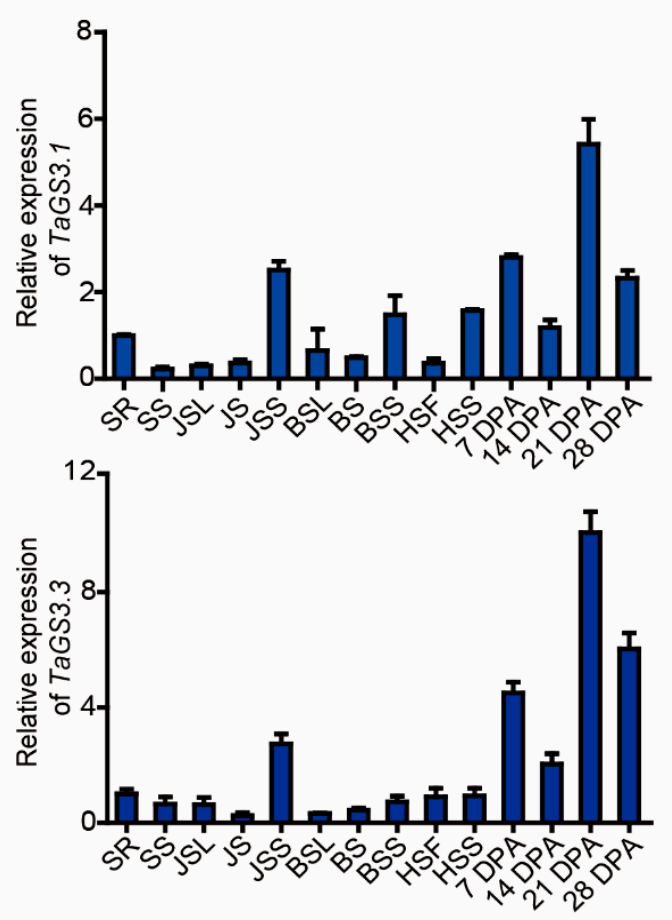

B

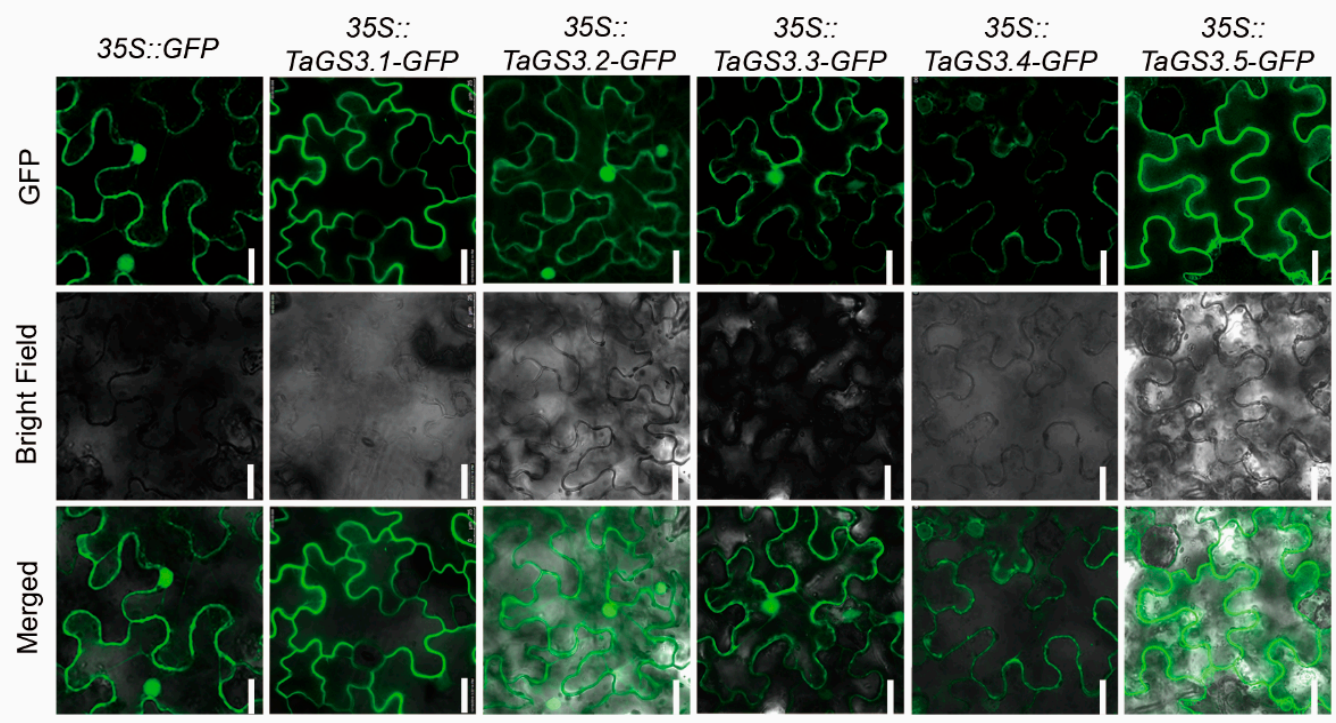

Figure 3. The expression patterns and subcellular localization of TaGS3 splicing variants. (A) Expression analyses of the TaGS3 splicing variants. SR, seedling root; SS, seedling shoot; JSL, leaves at jointing stage; JS, stems at jointing stage; JSS, spikes at jointing stage; BSL, leaves at booting stage; BS, stems at booting stage; BSS, spikes at booting stage; HSF, flag leaves at heading stage; HSS, spikes at heading stage; 7 DPA-28 DPA, grains at 7-28 days post-anthesis, respectively. Normalized expression values of TaGS3 splicing variants relative to GAPDH were given as mean \pm SEM from three replicates. (B) Subcellular localization of the TaGS3 isoforms. GFP and the five TaGS3 splicing variants fused with GFP under the control of the CaMV $35 S$ promoter were transiently expressed in epidermal cells of N. benthamiana. Bar $=25 \mu \mathrm{m}$. 
A

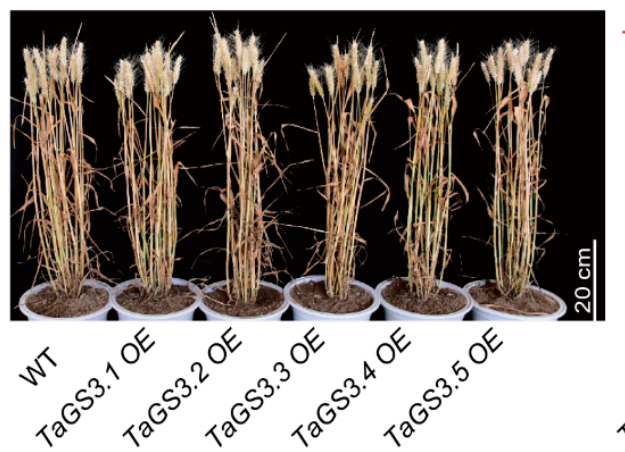

C

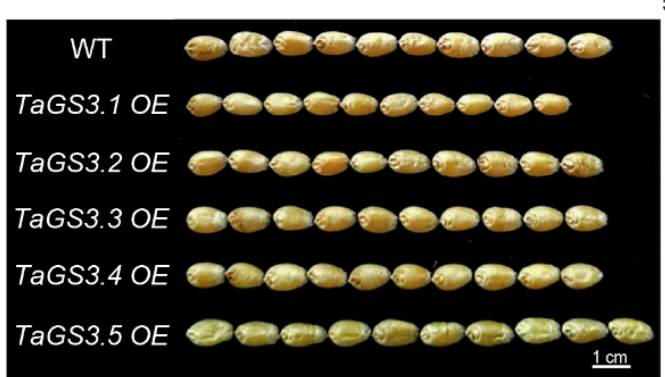

D

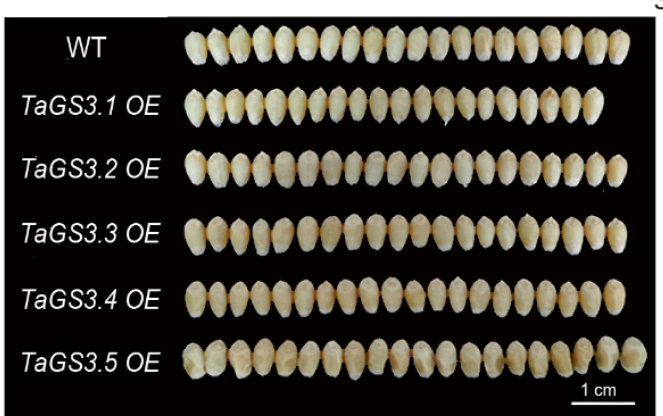

E

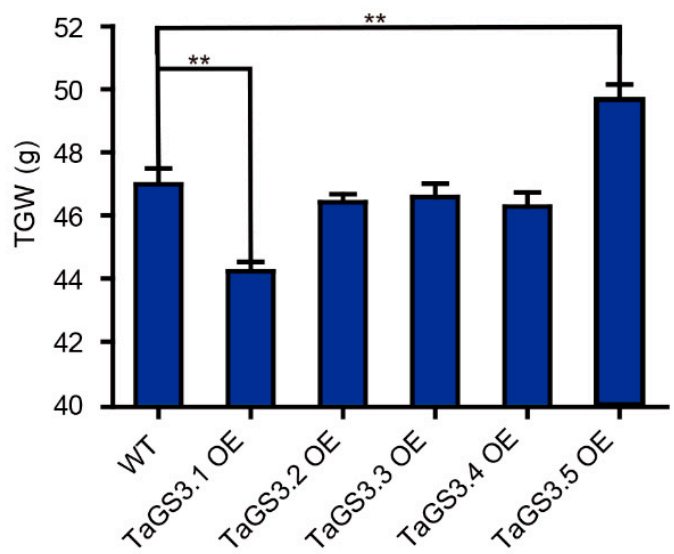

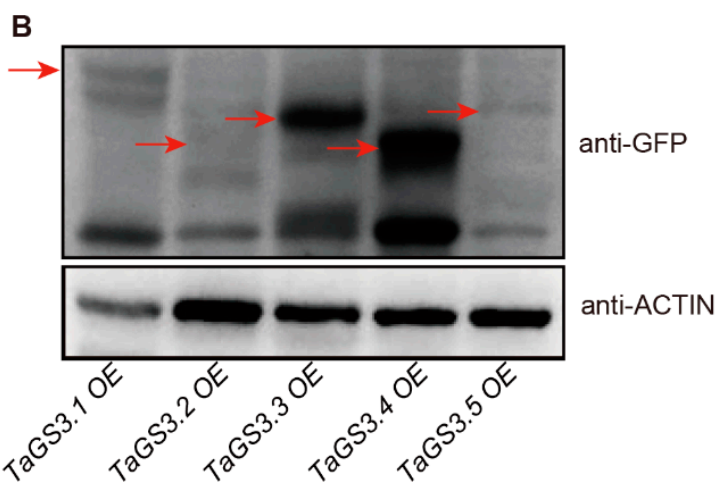

$\mathrm{GL}(\mathrm{mm})$
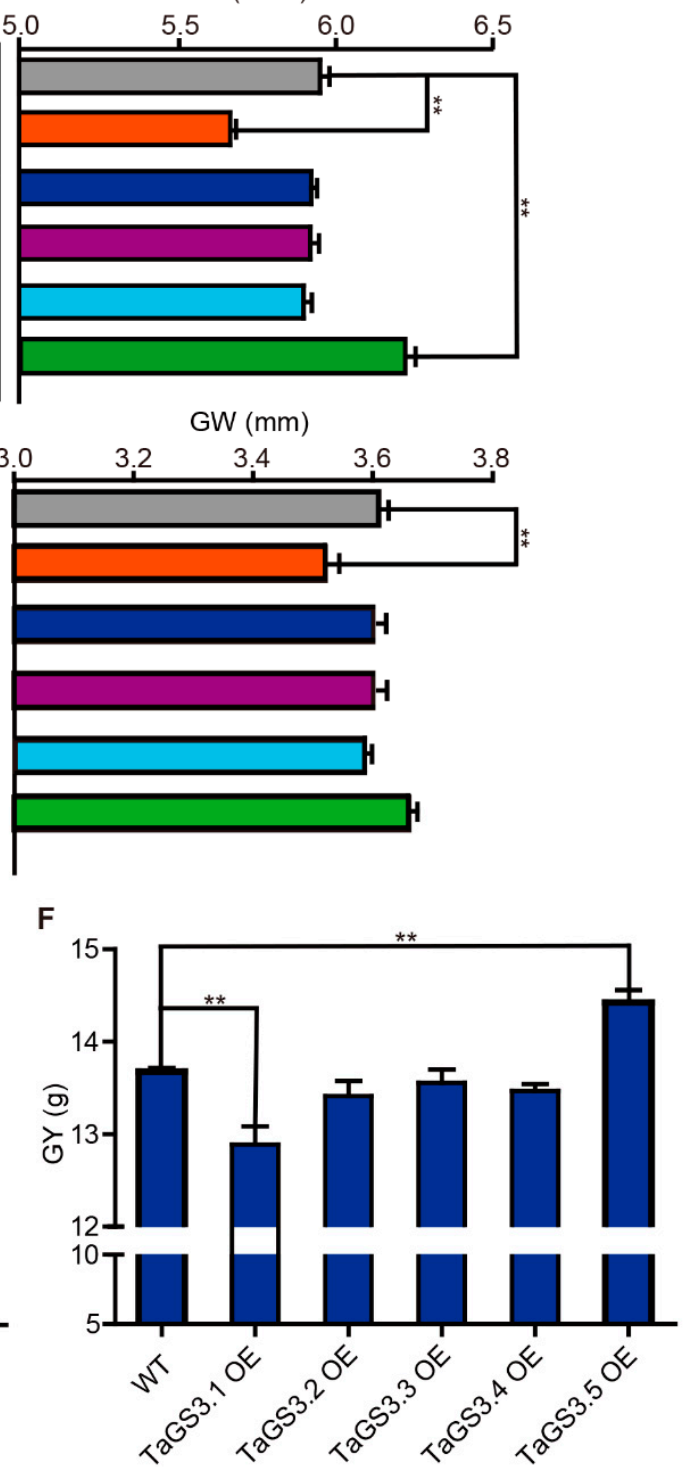

Figure 4. Phenotypic comparison of agronomic attributes among the overexpression lines of TaGS3 splicing variants and WT under field conditions. (A) Overview of the whole plants of TaGS3 splicing variants overexpression lines and WT. Bar $=20 \mathrm{~cm}$. (B) Immunoblot analysis of the $T_{3}$ transgenic plants of TaGS3 splicing variants. Total protein was extracted from the leaves of 3-day-old seedlings for Western blotting. (C) Comparison of the grain length among the tested genotypes. Bar $=1 \mathrm{~cm}$. (D) Comparison of the grain width among the tested genotypes. Bar $=1 \mathrm{~cm}$. (E) Thousand-grain weight (TGW) and (F) grain yield per plant (GY) of the tested genotypes. ${ }^{* *} p<0.01$ (Tukey test) indicates significant differences between the overexpression lines and WT. Data are given as mean \pm SEM $(n=10)$. 
The agronomic attributes of TaGS3.1-3.5 overexpression lines were examined in field studies conducted in 2019. No significant differences were found between TaGS3.1-3.5 overexpression lines and WT in terms of plant height $(\mathrm{PH})$, spike number per plant (SN), spike length (SL), total spikelets per spike (TS), sterile spikelets per spike (SS), and grain number per spike (GN) (Figure 4A; Table S2). However, significant differences were detected for each TaGS3.1-3.5 overexpression line compared with WT in grain traits such as grain length (GL), grain width (GW), thousand-grain weight (TGW), and grain yield per plant (GY) (Figure $4 \mathrm{C}-\mathrm{F}$ ). Compared to WT, TaGS3.1 overexpression lines exhibited significantly smaller and lighter grains, with a reduction of 5.04\% (0.30 $\pm 0.04 \mathrm{~mm})$ in GL, $2.49 \%(0.08 \pm 0.02 \mathrm{~mm})$ in GW, 5.89\% (2.77 $\pm 0.54 \mathrm{~g})$ in TGW, and 5.77\% (0.79 $\pm 0.19 \mathrm{~g})$ in GY. There were no significant variations in GL, GW, TGW, and GY between TaGS3.2-3.4 overexpression lines and WT, but a $0.39-0.95 \%$ decrease in GL, $0.80-1.52 \%$ decrease in TGW and 0.92-1.91\% decrease in GY between TaGS3.2-3.4 overexpression lines and WT. Notably, TaGS3.5 overexpression lines produced significantly larger and heavier grains, with an increase of $4.39 \%(0.26 \pm 0.03 \mathrm{~mm})$ in GL, $1.39 \%(0.05 \pm 0.02 \mathrm{~mm})$ in GW, 5.70\% $(2.68 \pm 0.65 \mathrm{~g})$ in TGW, and $5.41 \%(0.74 \pm 0.19 \mathrm{~g})$ in GY (Figure $4 \mathrm{C}-\mathrm{F})$. During three growing seasons, all TaGS3.1-3.5 overexpression lines displayed a recurring phenotype, similar to the results shown above (Table S2). These results suggested that TaGS3.1 and TaGS3.5 make opposing contributions to grain weight and grain size.

\subsection{TaGS3.1, TaGS3.2, TaGS3.3, and TaGS3.4 Physically Interact with WGB1}

The $G$ protein consists of $G_{\alpha}, G_{\beta}$ and $G_{\gamma}$ subunits, where $G_{\alpha}$ and $G_{\beta}$ play fundamental roles in the transduction of $G$ protein-mediated growth signals, and $G_{\beta}$-mediated signal transduction requires $G_{\gamma}$ for formation of active $G_{\beta \gamma}$ heterodimer [32,33]. To determine which section of TaGS3 is essential for $G_{\beta \gamma}$ dimer formation, we generated five TaGS3 isoforms: the full-length GS3 $3^{1-170}$ and the truncated $G S 3^{1-66}, G S 3^{1-60}, G S 3^{1-51}$, and $G S 3^{67-170}$. Different from $G S 3^{1-170}$, the $G S 3^{1-66}, G S 3^{1-60}$, and $G S 3^{1-51}$ isoforms were all devoid of the C-terminal Cys-rich region. GS3 ${ }^{1-66}$ contains an intact OSR domain, while GS3 ${ }^{1-60}$ contains an incomplete OSR domain lacking the six amino acid residues of the highly conserved DPFITI motif, and $G S 3^{1-51}$ contains an incomplete OSR domain that lacks 15 amino acid residues including the conserved DPFITI motif. By contrast, GS3 ${ }^{67-170}$ only comprises the C-terminal Cys-rich region without the N-terminal OSR domain (Figure 5A). Yeast two-hybrid and $\beta$-galactosidase assays demonstrated that GS3 ${ }^{1-170}, G S 3^{1-66}$, and GS3 ${ }^{1-60}$ interact with WGB1, in which $G S 3^{1-66}$ exhibits stronger affinity to WGB1 compared to $G S 3^{1-170}$, while GS3 ${ }^{1-60}$ shows weak affinity to WGB1, indicating that both the conserved DPFITI motif and the deletion of the C-terminal Cys-rich region are required for the enhancement of GS3 binding to WGB1. GS3 $3^{1-51}$ and GS3 $3^{67-170}$, on the other hand, did not interact with WGB1 (Figure 5B). These results suggest that GS3 interacts with WGB1 through the OSR domain rather than the C-terminal Cys-rich region, and the section of the 15 amino acid residues in the OSR domain is essential for the binding of GS3 to WGB1. 
A

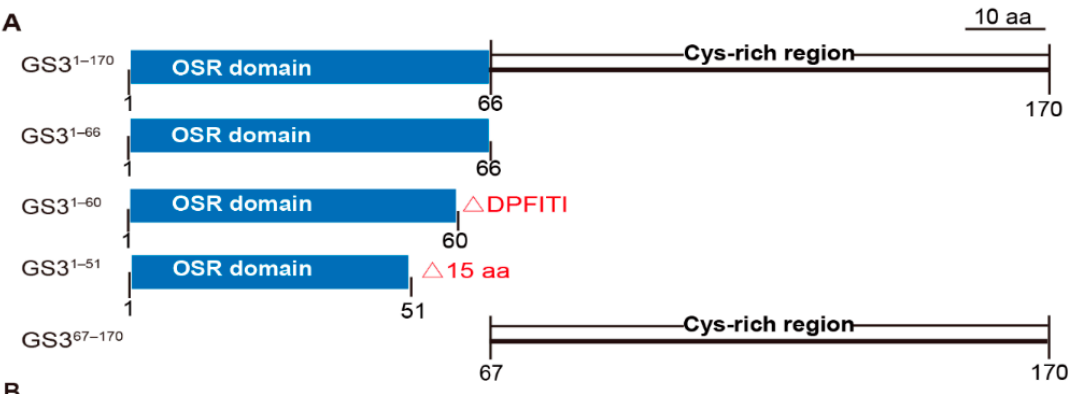

B

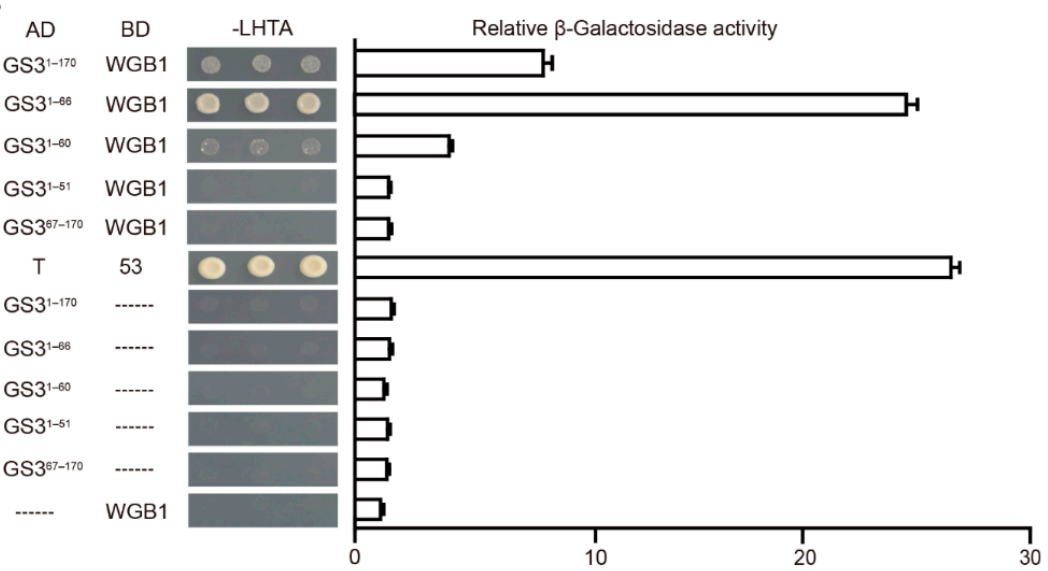

C

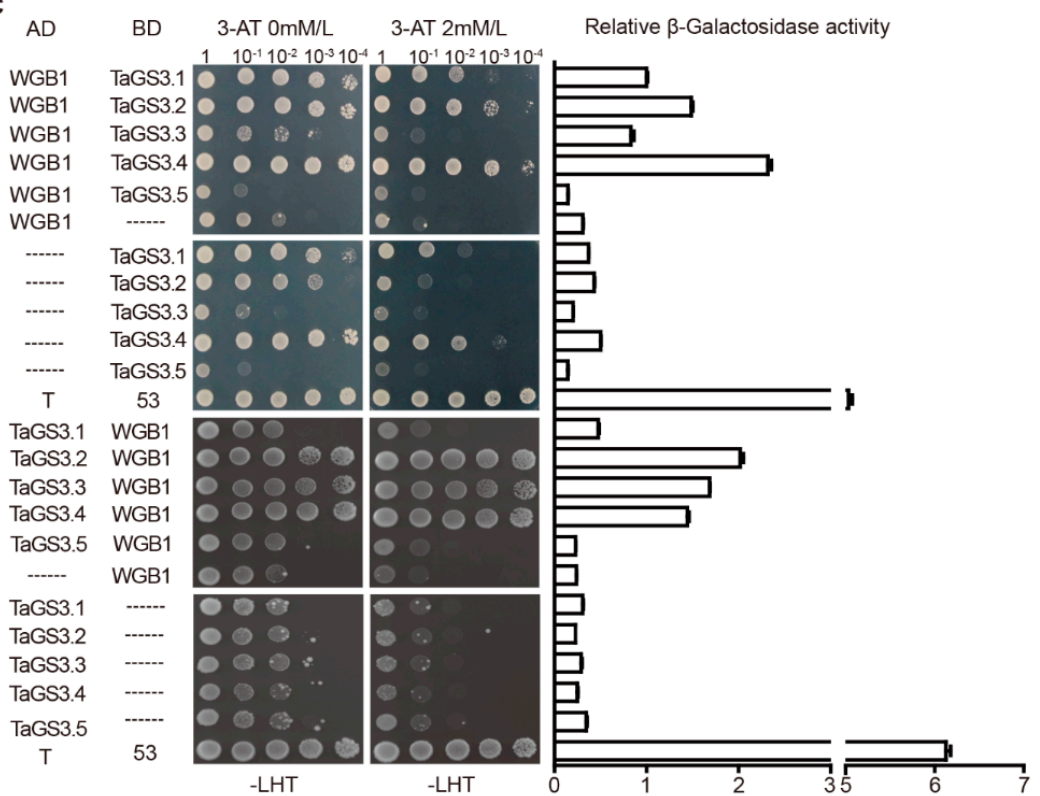

Figure 5. TaGS3 interacts with WGB1 through the OSR domain, rather than the C-terminal Cysrich region. (A) Schematic structure of protein versions with specific deleted domains. (B) Yeast two-hybrid assay showing the interactions of full-length $G S 3^{1-170}$ and the four truncated proteins $\left(G S 3^{1-66}, G S 3^{1-60}, G S 3^{1-51}\right.$, and GS3 ${ }^{67-170}$ ) with WGB1. AD, GAL4 activation domain; BD, GAL4 DNA binding domain; AD and BD represent empty pGADT7 and pGBKT7 vectors, respectively. AD-T/BD-53, positive control. -LHTA, selective medium lacking Leu, His, Trp and Ade. Quantitative analyses of interactions by $\beta$-galactosidase assay were shown (on the right) as mean \pm SEM $(n=3)$. (C) Yeast two-hybrid assay showing the interactions of TaGS3 isoforms with WGB1. -LHT, selective medium lacking Leu, His and Trp. The yeast strains were serially diluted $\left(\mathrm{OD}_{600}, 10^{-1}-10^{-4}\right)$ before spotting on selection medium-synthetic dropout interaction medium -LHT. The specificity of the stringency of the assay was tested by adding 3-aminotriazole (3-AT, $0 \mathrm{mM} / \mathrm{L}$ and $2 \mathrm{mM} / \mathrm{L}$, respectively). Quantitative analyses of interactions by $\beta$-galactosidase assay were shown (on the right) as mean $\pm \operatorname{SEM}(\mathrm{n}=3)$. 
Subsequently, we addressed whether the five TaGS3 isoforms encoded by TaGS3 splicing variants form a heterodimeric complex with WGB1. Here, the amino acid sequence of $T a G S 3.1$ was the same as $G S 3^{1-170}$. Yeast two-hybrid and $\beta$-galactosidase assays confirmed that TaGS3.2, TaGS3.3, and TaGS3.4 bind more tightly to WGB1 than TaGS3.1. TaGS3.5, lacking the 15 amino acid residue section in the OSR domain, does not bind to WGB1 (Figure 5). These results consistently support the necessity of the 15 amino acid residues in the OSR domain for the TaGS3-WGB1 interaction and the enhanced binding to WGB1 by TaGS3 when the C-terminal Cys-rich region is deleted. Furthermore, these results of the yeast two-hybrid assay were confirmed by pull-down and coimmunoprecipitation (Co-IP) assays, where WGB1 interacted with TaGS3.1, TaGS3.2, TaGS3.3, and TaGS3.4 instead of TaGS3.5 in vitro (Figure 6A-J).

A

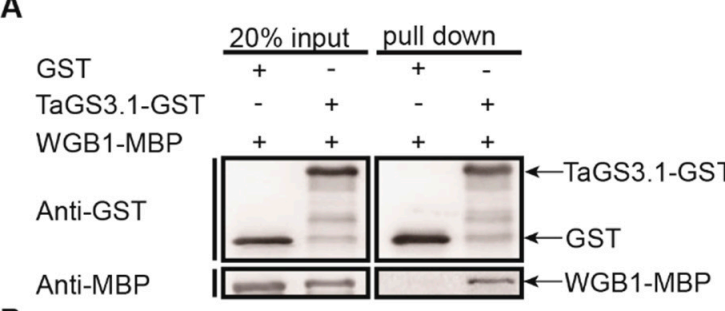

B

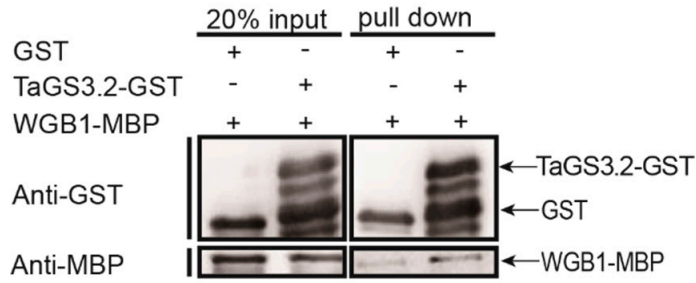

C

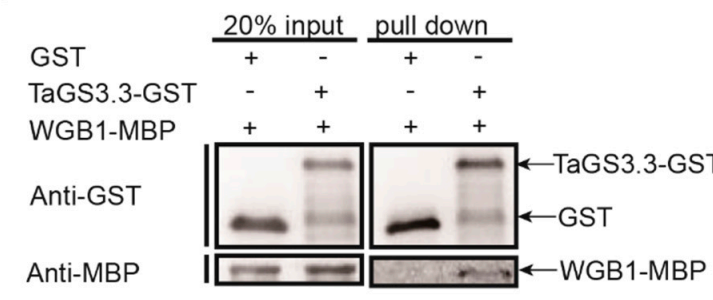

D

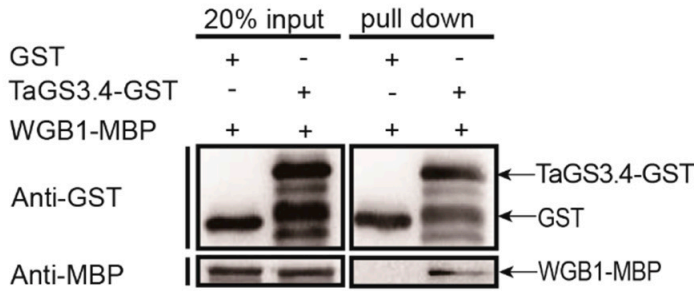

E

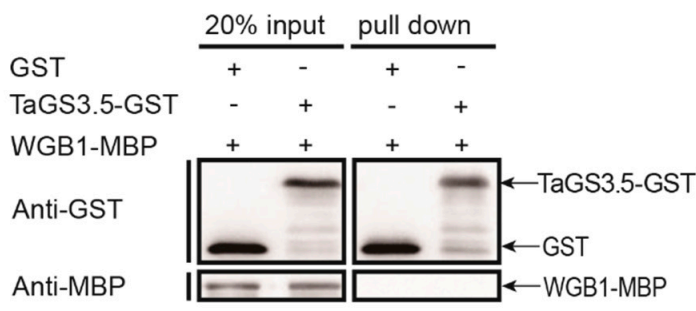

$\mathbf{F}$

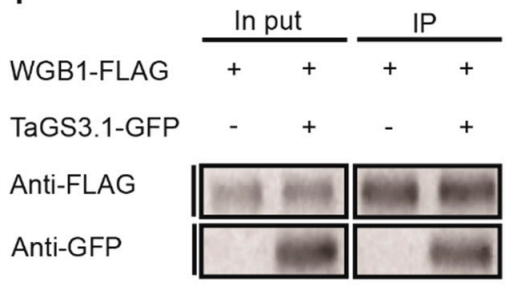

G

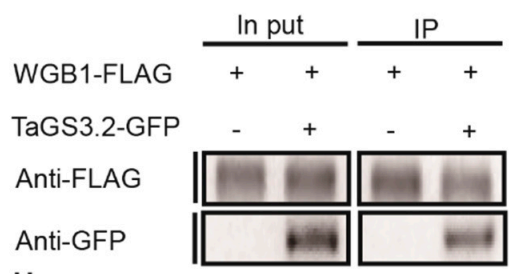

H

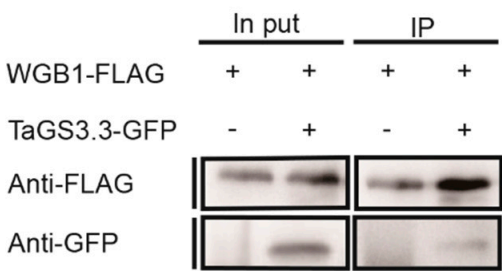

I

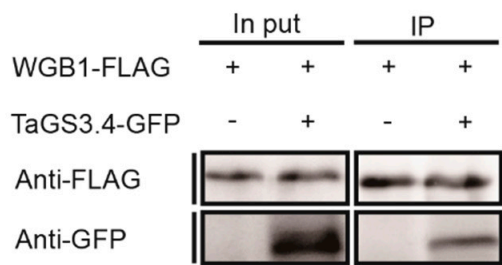

J

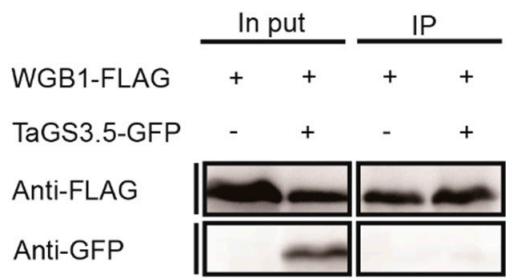

Figure 6. Interaction analyses of TaGS3 isoforms with WGB1. (A-E) In vitro GST pull-down assays showing the interactions between TaGS3 isoforms and WGB1. Recombinant maltose binding protein WGB1-MBP and fiveTaGS3 isoforms-GST were used. (F-J) In vivo Co-IP assays showing the interactions between TaGS3 isoforms and WGB1. FLAG-tagged WGB1 was co-expressed with GFP-tagged TaGS3 isoforms in N. benthamiana leaves. 


\subsection{Competitive Interactions of TaGS3 Isoforms with WGB1}

In a nonactivated state, the $\mathrm{G}_{\alpha}$ subunit maintains its GDP-bound state, forming an inactive heterotrimer with the $G_{\beta \gamma}$ dimer [34]. To test whether TaGS3 isoforms form a heterotrimer with WGB1 and WGA1, we performed yeast three-hybrid assay and found that TaGS3.1, TaGS3.2, TaGS3.3, and TaGS3.4, but not TaGS3.5, coupled with WGB1 to interact with WGA1 (Figure S8). In addition, yeast three-hybrid assay revealed competition between TaGS3.2-3.4 and TaGS3.1 in interaction with WGB1, where TaGS3.2-3.3 expression strongly disrupted the interaction of TaGS3.1 and WGB1, but TaGS3.4 expression slightly influenced interaction of TaGS3.1 and WGB1 (Figure 7). These results suggested competitive interactions between TaGS3.2-3.4 and TaGS3.1, providing an explanation for the functional existence and effects of multiple TaGS3 splicing variants for allowing fine-tuning of the regulation of wheat grain size.

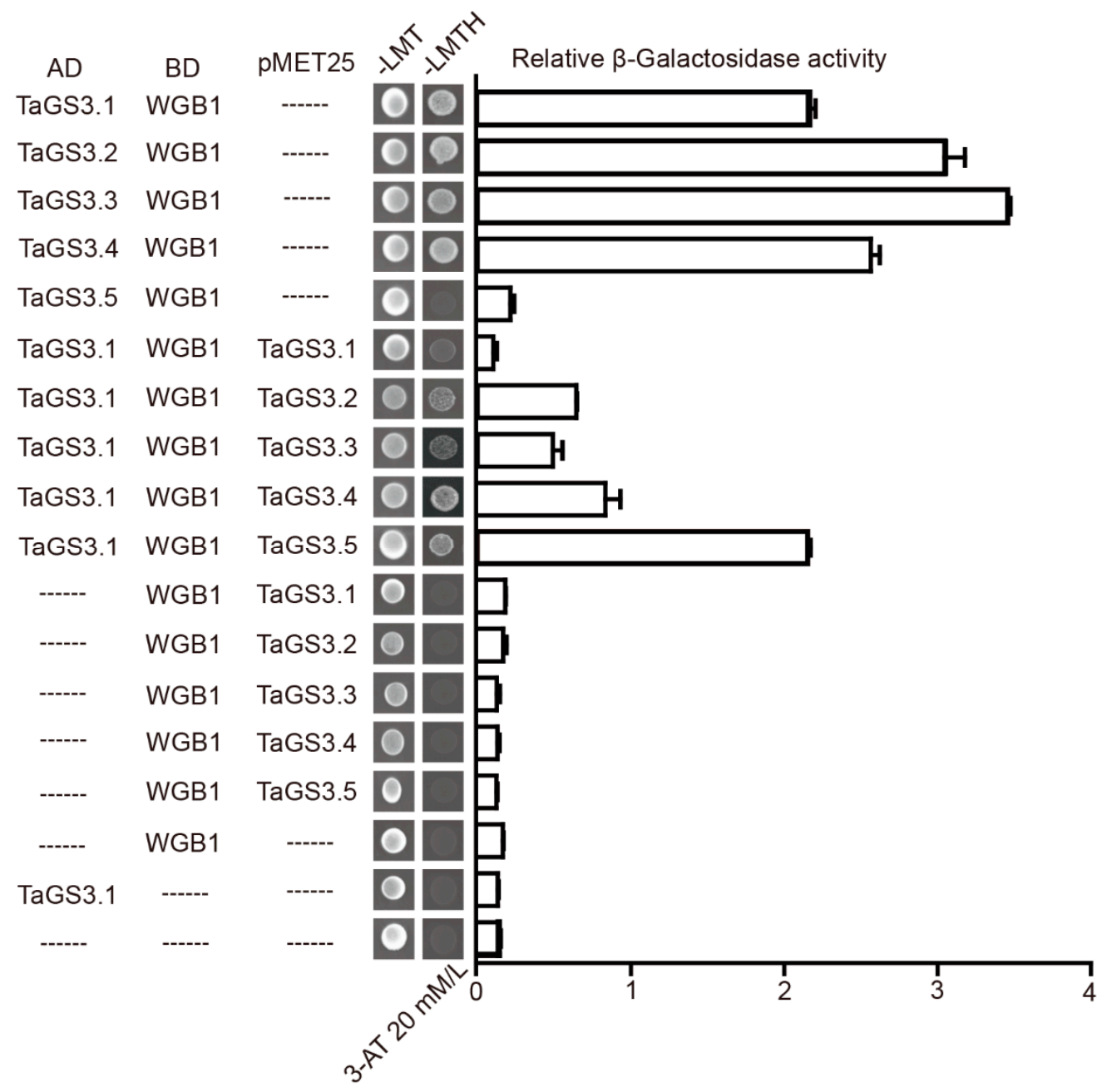

Figure 7. Competition test of the interactions between TaGS3 isoforms and WGB1. Yeast three-hybrid assay showing the competition between TaGS3.1 and the other four truncated TaGS3 isoforms to interact with WGB1 by using fusions with AD (AD-TaGS3 isoforms) and BD (BD-WGB1/BD-WGB1GS3 isoform) (on the left). AD and BD represent empty pGADT7 and pBridge vectors, respectively. Empty vector was used as the negative control. -LMT, selective medium lacking Leu, Met, and Trp; -LMTH, selective medium lacking Leu, Met, Trp, and His. The specificity of the stringency of the assay was tested by adding 3-aminotriazole (3-AT, $20 \mathrm{mM} / \mathrm{L}$ ). Quantitative analyses of interactions by $\beta$-galactosidase assay were shown (on the right) as mean $\pm \operatorname{SEM}(n=3)$. 


\section{Discussion}

\subsection{Conservation and Significance of GS3 AS in Gramineae}

Recent large-scale genomics studies, especially genome-wide analysis of gene expression profiling, revealed that $30 \%$ to $61 \%$ of genes underwent AS [35-37]. Considering the orthologous gene pairs in Arabidopsis and rice, 58\% of the same AS types were found, suggesting a role for the AS event as an evolutionarily conserved mechanism of posttranscriptional regulation [38]. In this study, five GS3 splicing variants were found to exist in both diploid and hexaploid Triticeae species (Figure 2C), indicative of the functional significance of ancestral candidate AS. The GS3.1, GS3.2, and GS3.3 variants occur in B. distachyon and GS3.1 and GS3.5 in barley (Figure S9 and Table S3), suggesting that AS of GS3 is conserved in wheat, barley, and B. distachyon. The constitutive variant GS3.1 is a major variant in both diploid ancestor grasses and hexaploid wheat, portraying a similar situation to most AS events in Arabidopsis and cereal crops, in that only one major transcript exists despite the presence of multiple splicing variants [14,39]. The frequency of AS occurrence in wheat tends to decrease after polyploidization, as determined by highthroughput transcriptome sequencing [24]. GS3 AS occurrence tends to decrease along with the increasing degree of wheat polyploidization, since a higher frequency of GS3 splicing variants is found in diploid wheat progenitor species compared with hexaploid wheat (Figure 2C).

Polyploidization is often accompanied by changes in genomic structure and gene expression [40]. In hexaploid KN9204 and KN199, a single nucleotide difference causes the absence of one canonical splice junction (AG) in the third intron of TaGS3-7A (Figure S5), which results in the third intron of TaGS3-7A differing from that of homeologs TaGS3-4A and TaGS3-7D. As a consequence, TaGS3.2 could not be isolated from TaGS3-7A in KN9204 and KN199. In our previous reports, TaGS3-4A and TaGS3-7D exhibited increased transcription abundance compared with TaGS3-7A in developing grains, suggesting that TaGS3-4A and $T a G S 3-7 D$ play more important roles in grain development [11]. Whether the presence of TaGS3.2 contributes to increased TaGS3 expression levels needs further investigation. Our findings here indicate that genomic-structure-triggered AS could contribute to functional diversity among homeologs in bread wheat, which is different from those caused by epigenetic modifications [41-43]. Grain size is a complex trait, and different levels of gene function regulation may dominate grain development. Therefore, we inferred the functional significance of TaGS3 AS event as an evolutionarily conserved mechanism in the regulation of wheat grain weight and size.

\subsection{Differential Functions and Mechanisms of TaGS3 ASs in Wheat}

Some splicing variants compete with the constitutive variant and interfere with its function in a dominant negative manner [17,44]. For instance, FLOWERING LOCUS T (FT) gene $F T 2$ generates two splicing variants in $B$. distachyon, $F T 2 \alpha$ and $F T 2 \beta$, which play antagonistic roles in regulating the flowering processes [15]. A similar mechanism was identified here. TaGS3.2, TaGS3.3, and TaGS3.4 compete with the constitutive TaGS3.1 variant in binding to WGB1 to form a functional $G_{\beta \gamma}$ heterodimer, respectively (Figures 6 and 7), and thereby regulate grain weight and size. However, the overexpression of TaGS3.2-3.4 that contain an intact OSR domain coding region (Figure 1B) did not obviously affect grain size (Figure 4C-E), which may be attributed to the low proportion of TaGS3.2-3.4 and their instability (Figure S7). On the other hand, there is abundant steady-state accumulation of TaGS3.3-GFP and TaGS3.4-GFP in overexpression lines (Figure 4B, Figure S7). We observed ectopic subcellular localization of TaGS3.3-GFP and TaGS3.4-GFP in the cytoplasm and nucleus (Figure 3B), with possible traces of functional TaGS3.3 and TaGS3.4 localized at the plasma membrane, consistent with the lack of obvious changes in the grain-size phenotype of TaGS3.3 and TaGS3.4 overexpression lines. Thus, given the stronger affinity of TaGS3.2-3.4 to WGB1 (Figure 5C), TaGS3.2-3.4 functions in antagonistically fine-tuning TaGS3.1 function. 
Furthermore, AS of FLM (FLOWERING LOCUS M) and HAB1 (HYPERSENSITIVE to ABA1) in Arabidopsis exhibited opposite effects by antagonistic splicing variants, respectively [17,44]. In this study, overexpressing TaGS3.5 significantly increased grain weight and size, which was opposite to the negative effect of TaGS3.1 (Figure 4). In the TaGS3.5 transgenic lines, TaGS3.5 expression level was markedly increased, while TaGS3.1 expression level was nearly unchanged (Figure S10), indicating that the positive effect of TaGS3.5 in enlarging grain size is independent of the TaGS3.1 expression level. TaGS3.5 is unable to interact with WGB1 to form active $\mathrm{G}_{\beta \gamma}$ heterodimer (Figure 6), indicating that the opposing functions of TaGS3.5 and TaGS3.1 do not arise from competition. TaGS3.5 was observed in trace amounts compared to TaGS3.1 (Figure 2C) but appeared stable in vivo (Figure S7). Thus, it is reasonable that the constant retention of TaGS3.5 in overexpression lines may obstruct the normal G-protein-mediated signal transduction pathway and lead to the production of large grains.

\subsection{Functional Diversification of GS3 in Rice and Wheat}

OsGS3 overexpression in rice generated shorter plants with smaller grains and variable grain number [45]. TaGS3.1 and TaGS3.5 overexpression here had contrasting and overwhelming effects on morphological grain traits but did not significantly impact plant height and spike characteristics (Figure 4), indicating the functional specialization of TaGS3 in grain size regulation in wheat. Furthermore, the increased TGW resulting from TaGS3.5 overexpression consistently contributed to increased individual grain yield (Table S2), indicating the great value of TaGS3.5 in the genetic improvement of wheat aimed at elevating grain yield.

Nucleotide differences among rice varieties mainly occur in exon regions of OsGS3, which regulate grain traits by producing frameshift mutations that cause premature transcriptional termination [10]. Compared with OsGS3, sequence polymorphism in TaGS3 mainly occurs in intron regions [11], which results in AS rather than allelic variations in TaGS3. Both the truncated OsGS3 and TaGS3 function in the determination of grain size, with the truncated GS3 proteins generated in different ways, namely by allelic variations in rice but by AS in wheat. A nonsense mutation ( $\mathrm{C}$ to $\mathrm{A}$ ) in the second exon of OsGS3 in the Minghui 63 allele results in a PTC and, consequently, truncated OsGS3 devoid of 17 amino acid residues of the OSR domain and the entire C-terminal Cys-rich region, with the production of longer grains [9]. Overexpressing TaGS3.5 in wheat also leads to longer grains (Figure 4). Notably, TaGS3.5, which arises from skipping of the third exon, results in a truncated TaGS3.5 lacking 15 amino acid residues that include the DPFITI motif that is part of the OSR domain (Figure 1). These observations in both rice and wheat demonstrate that an intact OSR domain is necessary for GS3 to function as a negative regulator of grain size. The role of TaGS3.5 with the whole C-terminal Cys-rich region, which is deleted in the truncated OsGS3 in Minghui 63, illustrates the causal relationship between the deletion of the 15 amino acid residues in the OSR domain and the positive regulation of grain size, showing a new hierarchical regulation mechanism mediated by AS variation in wheat.

The C-terminal Cys-rich region (tail) is characteristic of the noncanonical $\mathrm{G} \gamma$ subunits unique to rice. Variations in the length of the $\mathrm{C}$-terminal tail of $\mathrm{G}_{\gamma}$ protein distinctly affect the final grain phenotype, in which the long-tailed $G_{\gamma}$ protein gives long grains in rice while the short-tailed and tailless $G_{\gamma}$ proteins give short grains [45]. Compared with the wildtype OsGS3, a 1 bp deletion carried by the Chuan 7 allele results in a truncated OsGS3 that retains the OSR and TM domains but with deletion of most sections of the C-terminal tail, resulting in the production of super short grains [10]. Contrary to this, overexpressing the long-tailed TaGS3.1 in wheat results in the production of short grains, but overexpressing the tailless TaGS3.2-3.4 results in medium-sized grains (Figure 4C-F), suggesting that the length of the C-terminal Cys-rich region in TaGS3 is negatively correlated with grain size in wheat. These observations in both rice and wheat indicate that the C-terminal Cys-rich region of OsGS3 and TaGS3 have divergent functions in regulating grain weight and size. 


\section{Materials and Methods}

\subsection{Plant Materials}

The developing grains of diploid wheat progenitor species TMB02 (T. boeoticum, AA), TH02 (Ae. sharonensis, SS) and Y199 (Ae. tauschii, DD) and H. vulgare cv. Morex, B. distachyon Bd21 and T. aestivum cv. Kenong 9204 (KN9204, Center for Agricultural Resources Research, Institute of Genetics and Developmental Biology, Chinese Academy of Sciences, Shijiazhuang, China) and Kenong 199 (KN199, Center for Agricultural Resources Research, Institute of Genetics and Developmental Biology, Chinese Academy of Sciences, Shijiazhuang, China) were used to analyze GS3 sequences as previously described [11].

\subsection{Isolation of GS3 Splicing Variants}

Three sets of primers from $5^{\prime}$ UTR (untranslated region) and $3^{\prime}$ UTR were used to amplify TaGS3 coding sequences from chromosomes 7A, 4A, and 7D. PCR products were separated by electrophoresis in agarose gels, and the target bands were purified and cloned into the pTOPO-Blunt Simple Vector (Genstar, Beijing, China), which was transformed into DH5 $\alpha$ using the heat-shock method (Transgen, Beijing, China).

One set of specific primers corresponding to the CDS (coding sequence) of TaGS3 was used to amplify the specific TaGS3 target DNA, the single colonies of which were identified by PCR to distinguish the corresponding sequences of TaGS3.1 (253 bp), TaGS3.2 (260 bp), TaGS3.3 (267 bp), TaGS3.4 (335 bp), and TaGS3.5 (208 bp). Positive clones were confirmed by sequencing. Each TaGS3 splicing variant was analyzed by alignments with the corresponding coding sequence. The number of positive clones in TMB02, TH02, and Y199 was 609, 484, and 932, respectively. In KN9204, the number of positive clones from chromosomes 7A, 4A, and 7D was 858, 803, and 932, respectively. In KN199, the number of positive clones from chromosomes 7A, 4A, and 7D was 787, 820, and 794, respectively.

The coding regions of $H v G S 3$ and BdGS3 were cloned from $H$. vulgare cv. Morex and $B$. distachyon $\mathrm{Bd} 21$, respectively, on the basis of the corresponding reference sequences of H. vulgare cv. Lasa Goumang (SDOW01000566) and B. distachyon Beauv (XM_014896470.2), the single colonies of which were identified by PCR. Positive clones were confirmed by sequencing. Each $H v G S 3$ and $B d G S 3$ splicing variant was analyzed by alignments with the corresponding coding sequence. The number of positive clones in HvGS3 and BdGS3 was 47 and 4, respectively. All the primers are listed in Table S4.

The coding sequences of TaGS3.1, TaGS3.2, TaGS3.3, TaGS3.4, and TaGS3.5 from chromosome 4A of KN9204 were subcloned into PJIT163, for expression driven by the ubiquitin promoter, to generate constructs of TaGS3.1 OE, TaGS3.2 OE, TaGS3.3 OE, TaGS3.4 OE, and TaGS3.5 OE, respectively. The constructs were transformed into immature embryos of T. aestivum $\mathrm{cv}$. KN199 by gene gun methods [46]. At least 20 independent $\mathrm{T}_{0}$ transgenic plants of each construct were identified by PCR.

\subsection{Phenotype Assessment}

Five to seven $\mathrm{T}_{3}$ homozygous overexpression lines of each TaGS3 splicing variant and WT were grown in the field at Luancheng Agro-Ecosystem Experimental Station, CAS, China. A random block design with three replications was performed in which each overexpression line was planted in a 2-row plot that was $2 \mathrm{~m}$ long with $25 \mathrm{~cm}$ row spacing and 40 seeds per row. Prior to harvest, 10 random plants in the central region of each plot in the main tiller were examined to determine their plant height $(\mathrm{PH})$, spike length (SL), and spike number per plant (SN), and 30 spikes were randomly sampled and examined for determination of the total spikelets per spike (TS), sterile spikelets per spike (SS), and grain number per spike (GN). After harvest, the individual grain yield (GY) of 10 representative plants was recorded, and grains were collected for phenotype assessment. Grain agricultural traits, including grain length (GL), grain width (GW), and thousand-grain weight (TGW), were measured using an SC-G multifunctional seed analyzer (Wanshen, Hangzhou, China). 


\subsection{Plasmid Construct}

The coding regions of WGA1 $\left(\mathrm{G}_{\alpha}\right)$ and WGB1 $\left(\mathrm{G}_{\beta}\right)$ were cloned from $\mathrm{KN} 9204$ on the basis of the corresponding reference sequences of WGA1 $\left(\mathrm{G}_{\alpha}\right)$ (MG748862.1) and WGB1 $\left(\mathrm{G}_{\beta}\right)\left(\mathrm{XM} \_037566621\right)$ in Chinese Spring (CS). For subcellular localization examination, the coding sequences of five TaGS3 splicing variants were cloned into the pCAMBIA 1300-35S-GFP vector. In the protein-protein interaction experiments, the coding sequences of the five TaGS3 splicing variants and DNA sequences to code the full-length GS3 ${ }^{1-170}$ and the truncated $G S 3^{1-66}, G S 3^{1-60}, G S 3^{1-51}$, and $G S 3^{67-170}$ were cloned into pGADT7; then, the coding sequence of WGB1 was cloned into the pGBKT7 vector for the yeast two-hybrid assay, and the coding sequences of the five TaGS3 splicing variants and WGB1 were cloned into pMAL-C2X and pGEX-4T-1, respectively, for the pull-down assay. For the coimmunoprecipitation assay, the coding sequences of the five TaGS3 splicing variants and WGB1 were cloned into the PCAMBIA1300-35S-GFP and pCAMBIA1300-35S-FLAG vectors, respectively. For the yeast three-hybrid assay, the coding sequences of WGA1 were cloned into pGADT7, and the coding sequences of WGB1 and five TaGS3 splicing variants were cloned into the $\mathrm{pBridge}$ vector. All primers for vector construction are listed in Table S4.

\subsection{RNA Extraction and $q R T-P C R$}

For tissue-specific expression analysis, different leaf tissues from the plant at the seedling, jointing, booting, and heading stages, along with grain samples of 7, 14, 21, and 28 days post-anthesis were collected from the overexpression lines of the five TaGS3 splicing variants grown in the field. Total RNA was isolated using an RNA extraction kit (Tiangen, Beijing, China) and quantified by Nanodrop (Thermo, Waltham, MA, USA). First-strand cDNA was synthesized from DNaseI-treated total RNA using the PrimeScript RT Reagent Kit (TaKaRa, Tykyo, Japan) according to the manufacturer's instructions.

qRT-PCR was carried out in a total volume of $20 \mu \mathrm{L}$ using the SYBR PCR kit (TaKaRa, Tykyo, Japan) and on a CFX96 Real-Time PCR Detection System (Bio-Rad, Hercules, CA, USA) according to the manufacturer's instructions. At least three biological replicates were assayed, for which three technical replications were conducted. The GAPDH locus served as a normalization control to determine the relative expression level of each splicing variant. Specific primer sequences are listed in Table S4.

\subsection{Subcellular Localization Assay}

The 35S::TaGS3.1-GFP, 35S::TaGS3.2-GFP, 35S::TaGS3.3-GFP, 35S::TaGS3.4-GFP, and 35S::TaGS3.5-GFP constructs were introduced into Agrobacterium tumefaciens strain GV3101pMP90, which was then transformed into epidermal cells of $N$. benthamiana. The empty 35S::GFP vector was used as the control. The transformed $N$. benthamiana leaves were cultivated for 48-72 $\mathrm{h}$ before sampling. Confocal imaging was conducted using laser scanning microscopy (Zeiss, Jena, Germany) according to the manufacturer's instructions.

\subsection{Yeast Two- and Three-Hybrid Assays}

The yeast two-hybrid experiment was performed using the MATCHMAKER GAL4 Two-Hybrid System (Clontech, Mountain View, CA, USA). Briefly, respective prey and bait vectors were cotransformed into yeast strain $\mathrm{AH} 109$ using the lithium acetate transformation method. The transformed yeast cells were selected on SD/-Leu-Trp (SD/-LT) dropout medium. Interactions were verified on the SD/-Leu-His-Trp (SD/-LHT) dropout medium with different concentrations of 3-aminotriazole $(0$ and $2 \mathrm{mM} / \mathrm{L})$.

For the yeast three-hybrid assay, prey vector pGADT7-WGA1 was co-transformed with different pBridge (Clontech, Mountain View, CA, USA) bait vectors TaGS3.1-WGB1, TaGS3.2-WGB1, TaGS3.3-WGB1, TaGS3.4-WGB1, and TaGS3.5-WGB1 into yeast strain AH109; prey vectors pGADT7-TaGS3.1, pGADT7-TaGS3.2, pGADT7-TaGS3.3, pGADT7TaGS3.4, and pGADT7-TaGS3.5 were co-transformed with pBridge (Clontech, Mountain View, CA, USA) bait vector WGB1-TaGS3.1 into yeast strain AH109. A single clone of 
AH109 from SD/-Met plate was grown on SD/-Leu-Met-Trp (SD/-LMT) medium, and interactions were verified on SD/-Leu-Met-Trp-His (SD/-LMTH) plates with 10 and $20 \mathrm{mM} / \mathrm{L}$ 3-aminotriazole.

For liquid $\beta$-galactosidase assay with ONPG as substrate, liquid cultures in SD/-LHT with three yeast colonies were inoculated overnight. The calorimetric $\beta$-galactosidase assay using the supernatant and the following activity calculation were performed as described in the Clontech Yeast Protocols Handbook.

\subsection{Pull-Down Assay}

TaGS3.1-GST, TaGS3.2-GST, TaGS3.3-GST, TaGS3.4-GST, TaGS3.5-GST, and WGB1-MBP plasmids were transformed into BL21 cells. Protein purification was performed using Glutathione Sepharose ${ }^{\mathrm{TM}} 4 \mathrm{~B}$ (GE Healthcare, Uppsala, Sweden) and Amylose Resin (NEB, Beverly, MA, USA) according to manufacturer instructions. GST beads were washed with GST binding buffer four times to remove the ethanol. Purified GST and TaGS3.1-GST, TaGS3.2-GST, TaGS3.3-GST, TaGS3.4-GST, and TaGS3.5-GST proteins were incubated with the same volume of GST beads in binding buffer (20 mM Tris- $\mathrm{HCl}(\mathrm{pH} 7.5), 150 \mathrm{mM}$ $\mathrm{NaCl}, 1 \%$ Nonidet P40, with $1 \mathrm{mM}$ phenylmethylsulfonyl fluoride (PMSF) and $0.5 \mathrm{mM}$ dithiothreitol) for $2 \mathrm{~h}$ at room temperature. These proteins were centrifuged at $800 \times g$ for $3 \mathrm{~min}$, and pellets were extensively washed with binding buffer four times to remove redundant proteins. Each purified protein was incubated with WGB1-MBP in binding buffer for another $2 \mathrm{~h}$ at room temperature or $4{ }^{\circ} \mathrm{C}$ overnight. Next, the mixture was washed four times by binding buffer to remove redundant WGB1-MBP. Samples were then collected and boiled for $5 \mathrm{~min}$ in $2 \times$ SDS protein loading buffer prior to Western blotting assay. The anti-MBP (TDY Biotech, Beijing, China, 1:5000) and anti-GST antibodies (TDY Biotech, Beijing, China, 1:5000) were used to detect MBP and GST tagged proteins, respectively.

\subsection{Coimmunoprecipitation Assay}

Leaves of 4- to 6-week-old N. benthamiana plants were agroinfiltrated with TaGS3.1GFP, TaGS3.2-GFP, TaGS3.3-GFP, TaGS3.4-GFP, TaGS3.5-GFP, and WGB1-FLAG when GFP served as control. After 2-day agroinfiltration, leaves were collected and ground to a fine powder in liquid nitrogen using a mortar and pestle. Total protein was then extracted with IP buffer (50 mM Na $2 \mathrm{HPO}_{4} / \mathrm{NaH}_{2} \mathrm{PO}_{4}$ (pH 7.4), $150 \mathrm{mM} \mathrm{NaCl}, 1 \%$ Triton X-100, $15 \%$ glycerol, $1 \mathrm{mM}$ phenylmethylsulfonyl fluoride (PMSF), and protease inhibitor cocktail (Roche)) and centrifuged at $14,000 \times g$ at $4{ }^{\circ} \mathrm{C}$ three times. Anti-FLAG M2 Affinity Gel (Sigma, St.louis, Mo, USA) was washed four times with PBS buffer and incubated with the extracted proteins at $4{ }^{\circ} \mathrm{C}$ for $3 \mathrm{~h}$ with gentle shaking. After centrifugation at $600 \times g$ and $4{ }^{\circ} \mathrm{C}$ for $1 \mathrm{~min}$, the precipitated samples were washed five times with washing buffer (50 $\mathrm{mM} \mathrm{Na}_{2} \mathrm{HPO}_{4} / \mathrm{NaH}_{2} \mathrm{PO}_{4}$ (pH 7.4), $150 \mathrm{mM} \mathrm{NaCl}, 0.1 \%$ Triton X-100, $10 \%$ glycerol, $1 \mathrm{mM}$ PMSF, and protease inhibitor cocktail (Roche)). The precipitate was suspended and boiled for $5 \mathrm{~min}$ in $2 \times$ SDS protein loading buffer, followed by SDS-PAGE electrophoresis for Western blotting. The anti-GFP (Abcam, Cambridge, UK, 1:2000) or anti-FLAG (Abcam, Cambridge, UK, 1:1000) antibody was used to examine the candidate protein.

\subsection{Protein Extraction and Immunoblotting Assay}

Total protein of 3-day-old seedlings was extracted using the extraction buffer (50 $\mathrm{mM}$ Tris, $\mathrm{pH} 7.5,150 \mathrm{mM} \mathrm{NaCl}, 0.1 \%$ Triton X-100, 0.2\% Nonidet P-40, protease inhibitor cocktail (Roche)). For the immunoblotting assay, the 3-day-old seedlings of TaGS3.1-GFP, TaGS3.2-GFP, TaGS3.3-GFP, TaGS3.4-GFP, and TaGS3.5-GFP transgenic plants were treated with proteasome inhibitor MG132 $(50 \mu \mathrm{M})$ and protein synthesis inhibitor CHX $(30 \mu \mathrm{M})$ for $0,6,12$, and $20 \mathrm{~h}$, respectively. The wheat shoot $(1 \mathrm{~g})$ was lysed in $800 \mu \mathrm{L}$ extraction buffer before centrifugation at $13,500 \times g$ for collection of the supernatant, which was boiled for $5 \mathrm{~min}$ in $2 \times$ SDS protein loading buffer for Western blotting. Each immunoblot was incubated with primary antibodies anti-GFP (Abcam, Cambridge, UK, 1:2000) and anti-ACTIN (Abcam, Cambridge, UK, 1:5000) for $1 \mathrm{~h}$ at room temperature or overnight 
at $4{ }^{\circ} \mathrm{C}$. Immunoblots were developed using peroxidase conjugated secondary antibody at antirabbit antibody (Abcam, Cambridge, UK, 1:2000) coupled to a chemiluminescence detection system. The obtained Western blot bands were quantified into their relative grey values using the ImageJ software (https:/ /imagej.nih.gov/ij/ accessed on 20 August 2021).

\subsection{Statistical Analyses}

Statistical analyses were based on phenotypic data for grain weight and size. One-way ANOVA was performed in the SPSS System for Windows version 20.0 (IBM Corporation, Armonk, NY, USA) to determine phenotypic differences between overexpression lines and WT, and Tukey tests were conducted to determine the significance of differences.

\subsection{Accession Numbers}

Sequence data from this study can be found in the GenBank database (http:/ / www. ncbi.nlm.nih.gov/ accessed on 15 August 2021) under the following accession numbers: HvGS3, SDOW01000566; BdGS3, XM_014896470; WGB1 $\left(\mathrm{G}_{\beta}\right)$ in Chinese Spring (CS), XM_037566621; WGA1 (G $\mathrm{G}_{\alpha}$ ) in Chinese Spring (CS), MG748862.1; HvGS3, KAE8784062.1; SiGS3, XP_004984061-1; SbGS3, XP_002465152.1; ZmGS3, NP_001354253.1; PhGS3, XP_025797819; OsGS3, XP_015630073.1; BdGS3.1, XP_014751956.

\section{Conclusions}

TaGS3 undergoes AS and produces five splicing variants that show differential functions in the determination of grain weight and size. AS of GS3 did not emerge during the process of wheat polyploidization, but pre-existed in the ancestor species of Triticeae crops. TaGS3.1 overexpression significantly reduced grain weight and length, TaGS3.2-3.4 overexpression did not significantly alter grain weight and size compared to wild type, and $T a G S 3.5$ overexpression significantly increased grain weight and grain length. TaGS3 isoforms with an intact OSR domain (TaGS3.1-3.4) interact with WGB1 to form $\mathrm{G}_{\beta \gamma}$ heterodimers that further interact with WGA1 to form inactive $G_{\alpha \beta \gamma}$ heterotrimers. Truncated isoforms TaGS3.2-3.4 lacking the C-terminal Cys-rich region showed enhanced binding affinity to WGB1 and antagonistically compete with TaGS3.1 to bind WGB1, while TaGS3.5 with the incomplete OSR domain does not interact with WGB1. Taken together, the results indicate that TaGS3 differentially regulates grain size via AS, by which the regulation of grain size is fine-tuned and balanced at the post-transcriptional level.

Supplementary Materials: The following are available online at https:/ / www.mdpi.com/article/10 $.3390 /$ ijms222111692/s1.

Author Contributions: W.Z., J.L. and L.L. planned the research project. L.Z., X.R., W.Z., L.L., Q.S., D.M. and L.G. carried out the experiments. W.Z., X.R., L.Z., N.Z., L.S. and J.J. conducted the field trials and collected phenotype data. W.Z., X.R., L.L., L.Z., A.B., X.L. and J.L. wrote and revised the manuscript. All authors have read and agreed to the published version of the manuscript.

Funding: This research was jointly supported by grants from Hebei Natural Science Foundation (C2021205013), the National Natural Science Foundation of China (31771784) and China Agriculture Research System of MOF and MARA (CARS-03).

Institutional Review Board Statement: Not applicable.

Informed Consent Statement: Not applicable.

Acknowledgments: We are grateful to Yongzhong Xing from Huazhong Agricultural University of China for his critical review of the manuscript.

Conflicts of Interest: The authors declare that they have no competing interest. 


\section{References}

1. Brisson, N.; Gate, P.; Gouache, D.; Charmet, G.; Oury, F.-X.; Huard, F. Why are wheat yields stagnating in Europe? A comprehensive data analysis for France. Field Crops Res. 2010, 119, 201-212. [CrossRef]

2. Li, N.; Xu, R.; Li, Y. Molecular Networks of Seed Size Control in Plants. Annu. Rev. Plant Biol. 2019, 70, 435-463. [CrossRef]

3. Schmidt, C.J.; Thomas, T.C.; Levine, M.A.; Neer, E.J. Specificity of G-protein $\beta$ and $\gamma$ subunit interaction. J. Biol. Chem. 1992, 267, 13807-13810. [CrossRef]

4. Trusov, Y.; Rookes, J.E.; Tilbrook, K.; Chakravorty, D.; Mason, M.G.; Anderson, D.; Chen, J.G.; Jones, A.M.; Botella, J.R. Heterotrimeric $\mathrm{G}$ protein gamma subunits provide functional selectivity in $\mathrm{G}$ beta gamma dimer signaling in Arabidopsis. Plant Cell 2007, 19, 1235-1250. [CrossRef]

5. Trusov, Y.; Chakravorty, D.; Botella, J.R. Diversity of heterotrimeric G-protein gamma subunits in plants. BMC Res. Notes 2012, 5, 608. [CrossRef] [PubMed]

6. Li, S.; Liu, Y.; Zheng, L.; Chen, L.; Li, N.; Corke, F.; Lu, Y.; Fu, X.; Zhu, Z.; Bevan, M.W.; et al. The plant-specific G protein gamma subunit AGG3 influences organ size and shape in Arabidopsis thaliana. New Phytol. 2012, 194, 690-703. [CrossRef]

7. Fan, C.; Xing, Y.; Mao, H.; Lu, T.; Han, B.; Xu, C.; Li, X.; Zhang, Q. GS3, a major QTL for grain length and weight and minor QTL for grain width and thickness in rice, encodes a putative transmembrane protein. Theor. Appl. Genet. 2006, 112, 1164-1171. [CrossRef] [PubMed]

8. Yu, H.; Lin, T.; Meng, X.; Du, H.; Zhang, J.; Liu, G.; Chen, M.; Jing, Y.; Kou, L.; Li, X.; et al. A route to de novo domestication of wild allotetraploid rice. Cell 2021, 184, 1156-1170.e14. [CrossRef]

9. Fan, C.; Yu, S.; Wang, C.; Xing, Y. A causal C-A mutation in the second exon of GS3 highly associated with rice grain length and validated as a functional marker. Theor. Appl. Genet. 2009, 118, 465-472. [CrossRef] [PubMed]

10. Mao, H.; Sun, S.; Yao, J.; Wang, C.; Yu, S.; Xu, C.; Li, X.; Zhang, Q. Linking differential domain functions of the GS3 protein to natural variation of grain size in rice. Proc. Natl. Acad. Sci. USA 2010, 107, 19579-19584. [CrossRef]

11. Zhang, W.; Li, H.F.; Zhi, L.Y.; Su, Q.N.; Liu, J.J.; Ren, X.L.; Meng, D.Y.; Zhang, N.; Ji, J.; Zhang, X.Y.; et al. Functional markers developed from TaGS3, a negative regulator of grain weight and size, for marker-assisted selection in wheat. Crop J. 2020, 8, 943-952. [CrossRef]

12. Nilsen, T.W.; Graveley, B.R. Expansion of the eukaryotic proteome by alternative splicing. Nature 2010, 463, 457-463. [CrossRef] [PubMed]

13. Gao, P.; Quilichini, T.D.; Zhai, C.; Qin, L.; Nilsen, K.T.; Li, Q.; Sharpe, A.G.; Kochian, L.V.; Zou, J.; Reddy, A.S.; et al. Alternative splicing dynamics and evolutionary divergence during embryogenesis in wheat species. Plant Biotechnol. J. 2021. [CrossRef]

14. Syed, N.H.; Kalyna, M.; Marquez, Y.; Barta, A.; Brown, J.W. Alternative splicing in plants-coming of age. Trends Plant Sci. 2012, 17, 616-623. [CrossRef] [PubMed]

15. Qin, Z.; Wu, J.; Geng, S.; Feng, N.; Chen, F.; Kong, X.; Song, G.; Chen, K.; Li, A.; Mao, L.; et al. Regulation of FT splicing by an endogenous cue in temperate grasses. Nat. Commun. 2017, 8, 14320. [CrossRef]

16. Quesada, V.; Macknight, R.; Dean, C.; Simpson, G.G. Autoregulation of FCA pre-mRNA processing controls Arabidopsis flowering time. EMBO J. 2003, 22, 3142-3152. [CrossRef] [PubMed]

17. Pose, D.; Verhage, L.; Ott, F.; Yant, L.; Mathieu, J.; Angenent, G.C.; Immink, R.G.; Schmid, M. Temperature-dependent regulation of flowering by antagonistic FLM variants. Nature 2013, 503, 414-417. [CrossRef]

18. Seo, P.J.; Park, M.J.; Lim, M.H.; Kim, S.G.; Lee, M.; Baldwin, I.T.; Park, C.M. A self-regulatory circuit of CIRCADIAN CLOCKASSOCIATED1 underlies the circadian clock regulation of temperature responses in Arabidopsis. Plant Cell 2012, 24, 2427-2442. [CrossRef]

19. Howitt, C.A.; Cavanagh, C.R.; Bowerman, A.F.; Cazzonelli, C.; Rampling, L.; Mimica, J.L.; Pogson, B.J. Alternative splicing activation of cryptic exons and amino acid substitutions in carotenoid biosynthetic genes are associated with lutein accumulation in wheat endosperm. Funct. Integr. Genomics 2009, 9, 363-376. [CrossRef]

20. Cyrek, M.; Fedak, H.; Ciesielski, A.; Guo, Y.; Sliwa, A.; Brzezniak, L.; Krzyczmonik, K.; Pietras, Z.; Kaczanowski, S.; Liu, F.; et al. Seed dormancy in Arabidopsis is controlled by alternative polyadenylation of DOG1. Plant Physiol. 2016, 170, 947-955. [CrossRef]

21. Yu, J.; Miao, J.; Zhang, Z.; Xiong, H.; Zhu, X.; Sun, X.; Pan, Y.; Liang, Y.; Zhang, Q.; Abdul Rehman, R.M.; et al. Alternative splicing of OsLG3b controls grain length and yield in japonica rice. Plant Biotechnol. J. 2018, 16, 1667-1678. [CrossRef] [PubMed]

22. Pan, Y.H.; Gao, L.J.; Liang, Y.T.; Zhao, Y.; Liang, H.F.; Chen, W.W.; Yang, X.H.; Qing, D.J.; Gao, J.; Wu, H.; et al. OrMKK3 Influences Morphology and Grain Size in Rice. J. Plant Biol. 2021, 1-14. [CrossRef]

23. Brenchley, R.; Spannagl, M.; Pfeifer, M.; Barker, G.L.; D’Amore, R.; Allen, A.M.; McKenzie, N.; Kramer, M.; Kerhornou, A.; Bolser, D.; et al. Analysis of the bread wheat genome using whole-genome shotgun sequencing. Nature 2012, 491, 705-710. [CrossRef] [PubMed]

24. Yu, K.; Feng, M.; Yang, G.; Sun, L.; Qin, Z.; Cao, J.; Wen, J.; Li, H.; Zhou, Y.; Chen, X.; et al. Changes in alternative splicing in response to domestication and polyploidization in Wheat. Plant Physiol. 2020, 184, 1955-1968. [CrossRef]

25. Sun, Y.; He, Z.; Ma, W.; Xia, X. Alternative splicing in the coding region of $P p o-A 1$ directly influences the polyphenol oxidase activity in common wheat (Triticum aestivum L.). Funct. Integr. Genomics 2011, 11, 85-93. [CrossRef] [PubMed]

26. Zhou, Y.J.; Yang, Q.; Zhong, X.J.; Tang, H.P.; Deng, M.; Ma, J.; Qi, P.F.; Wang, J.R.; Chen, G.Y.; Liu, Y.X.; et al. Alternative splicing results in a lack of starch synthase IIa-D in Chinese wheat landrace. Genome 2018, 61, 201-208. [CrossRef] 
27. Zhang, H.; Mao, R.; Wang, Y.; Zhang, L.; Wang, C.; Lv, S.; Liu, X.; Wang, Y.; Ji, W. Transcriptome-wide alternative splicing modulation during plant-pathogen interactions in wheat. Plant Sci. 2019, 288, 110160. [CrossRef]

28. Egawa, C.; Kobayashi, F.; Ishibashi, M.; Nakamura, T.; Nakamura, C.; Takumi, S. Differential regulation of transcript accumulation and alternative splicing of a DREB2 homolog under abiotic stress conditions in common wheat. Genes Genet. Syst. 2006, 81, 77-91. [CrossRef]

29. Liu, Z.; Qin, J.; Tian, X.; Xu, S.; Wang, Y.; Li, H.; Wang, X.; Peng, H.; Yao, Y.; Hu, Z.; et al. Global profiling of alternative splicing landscape responsive to drought, heat and their combination in wheat (Triticum aestivum L.). Plant Biotechnol. J. 2018, 16, 714-726. [CrossRef]

30. Lu, Y.; Zhao, P.; Zhang, A.; Ma, L.; Xu, S.; Wang, X. Alternative splicing diversified the heat response and evolutionary strategy of conserved heat shock protein 90s in Hexaploid Wheat (Triticum aestivum L.). Front Genet. 2020, 11, 577897. [CrossRef]

31. Gracheva, E.O.; Cordero-Morales, J.F.; Gonzalez-Carcacia, J.A.; Ingolia, N.T.; Manno, C.; Aranguren, C.I.; Weissman, J.S.; Julius, D. Ganglion-specific splicing of TRPV1 underlies infrared sensation in vampire bats. Nature 2011, 476, 88-91. [CrossRef] [PubMed]

32. Temple, B.R.; Jones, A.M. The plant heterotrimeric G-protein complex. Annu. Rev. Plant Biol. 2007, 58, 249-266. [CrossRef] [PubMed]

33. Ullah, H.; Chen, J.G.; Young, J.C.; Im, K.H.; Sussman, M.R.; Jones, A.M. Modulation of cell proliferation by heterotrimeric G protein in Arabidopsis. Science 2001, 292, 2066-2069. [CrossRef] [PubMed]

34. Urano, D.; Jones, A.M. Heterotrimeric G protein-coupled signaling in plants. Annu. Rev. Plant Biol. 2014, 65, 365-384. [CrossRef]

35. Klepikova, A.V.; Kasianov, A.S.; Gerasimov, E.S.; Logacheva, M.D.; Penin, A.A. A high resolution map of the Arabidopsis thaliana developmental transcriptome based on RNA-seq profiling. Plant J. 2016, 88, 1058-1070. [CrossRef]

36. Marquez, Y.; Brown, J.W.; Simpson, C.; Barta, A.; Kalyna, M. Transcriptome survey reveals increased complexity of the alternative splicing landscape in Arabidopsis. Genome Res. 2012, 22, 1184-1195. [CrossRef]

37. Reddy, A.S.; Marquez, Y.; Kalyna, M.; Barta, A. Complexity of the alternative splicing landscape in plants. Plant Cell 2013, 25, 3657-3683. [CrossRef]

38. Wang, B.B.; Brendel, V. Genome wide comparative analysis of alternative splicing in plants. Proc. Natl. Acad. Sci. USA 2006, 103, 7175-7180. [CrossRef]

39. Wang, B.; Regulski, M.; Tseng, E.; Olson, A.; Goodwin, S.; McCombie, W.R.; Ware, D. A comparative transcriptional landscape of maize and sorghum obtained by single-molecule sequencing. Genome Res. 2018, 28, 921-932. [CrossRef]

40. Wendel, J.F. Genome evolution in polyploids. Plant Mol. Biol. 2000, 42, 225-249. [CrossRef]

41. Hu, Z.; Han, Z.; Song, N.; Chai, L.; Yao, Y.; Peng, H.; Ni, Z.; Sun, Q. Epigenetic modification contributes to the expression divergence of three TaEXPA1 homoeologs in hexaploid wheat (Triticum aestivum). New Phytol. 2013, 197, 1344-1352. [CrossRef]

42. Shitsukawa, N.; Tahira, C.; Kassai, K.; Hirabayashi, C.; Shimizu, T.; Takumi, S.; Mochida, K.; Kawaura, K.; Ogihara, Y.; Murai, K. Genetic and epigenetic alteration among three homoeologous genes of a class E MADS box gene in hexaploid wheat. Plant Cell 2007, 19, 1723-1737. [CrossRef] [PubMed]

43. Zhang, W.; Fan, X.; Gao, Y.; Liu, L.; Sun, L.; Su, Q.; Han, J.; Zhang, N.; Cui, F.; Ji, J.; et al. Chromatin modification contributes to the expression divergence of three TaGS2 homoeologs in hexaploid wheat. Sci. Rep. 2017, 7, 44677. [CrossRef]

44. Wang, Z.; Ji, H.; Yuan, B.; Wang, S.; Su, C.; Yao, B.; Zhao, H.; Li, X. ABA signalling is fine-tuned by antagonistic HAB1 variants. Nat. Commun. 2015, 6, 8138. [CrossRef] [PubMed]

45. Sun, S.; Wang, L.; Mao, H.; Shao, L.; Li, X.; Xiao, J.; Ouyang, Y.; Zhang, Q. A G-protein pathway determines grain size in rice. Nat. Commun. 2018, 9, 851. [CrossRef] [PubMed]

46. Zhang, K.; Liu, J.; Zhang, Y.; Yang, Z.; Gao, C. Biolistic genetic transformation of a wide range of Chinese elite wheat (Triticum aestivum L.) varieties. J. Genet. Genomics 2015, 42, 39-42. [CrossRef] [PubMed] 